\title{
Photodissociation of alkyl iodides in solution: Substituent effects on the early-time dynamics
}

\author{
David L. Phillips and Anne B. Myers ${ }^{\text {a) }}$ \\ Department of Chemistry, University of Rochester, Rochester, New York 14627
}

(Received 13 December 1990; accepted 28 March 1991)

\begin{abstract}
Resonance Raman spectra, including absolute scattering cross sections, have been measured for ethyl, isopropyl, and tert-butyl iodides in cyclohexane solution at seven to ten wavelengths between 303 and $200 \mathrm{~nm}$. Spectra of fully deuterated ethyl iodide have also been obtained at five wavelengths. Spectra excited in the 300-250 nm region, on resonance with the directly dissociative $A$ state, are dominated by long overtone progressions in the nominal $\mathrm{C}-\mathrm{I}$ stretching mode near $500 \mathrm{~cm}^{-1}$. In all three molecules the fundamental of the $\mathrm{C}-\mathrm{I}$ stretch is unexpectedly weak relative to the overtones when excited near the peak of the $A$ band. This is shown to arise from interference between the $A$-state resonant part of the fundamental Raman amplitude and preresonant contributions from higher electronic states. In addition to the $\mathrm{C}-\mathrm{I}$ stretching activity, $\boldsymbol{A}$-state excitation generates significant intensity in fundamentals, overtones, and combination bands of modes nominally assigned as bending and $\mathrm{CC}$ stretching vibrations, suggesting a multidimensional character to the reaction coordinate. The absorption spectra and $A$-state resonant Raman intensities are modeled successfully through wave-packet propagation on a multidimensional locally harmonic potential with a preresonant contribution to the fundamental intensities included. The short-time photodissociation dynamics are then examined by using the normal-mode coefficients to convert the wave-packet motion from dimensionless normal coordinates into internal coordinates. It is found that while the dominant motion during the first 10 fs involves stretching of the $\mathrm{C}-\mathrm{I}$ bond, other stretching and bending motions are also involved, although the precision of these conclusions for isopropyl and tert-butyl iodides is limited by the indeterminacy in the signs of the normalmode displacements obtained from the intensity analysis. Comparison of the results for normal and perdeuterated ethyl iodide is used to resolve most of the sign indeterminacies for this molecule. The present results are compared and contrasted to conclusions of previous studies of energy partitioning in the vapor-phase photodissociation.
\end{abstract}

\section{INTRODUCTION}

The alkyl iodides have been widely treated as a paradigm for photodissociation processes occurring on a directly repulsive excited-state potential surface. Photoexcitation of an alkyl iodide (RI) within its lowest electronic absorption band near $260 \mathrm{~nm}$ leads, at least in the vapor phase, to prompt R-I bond fission to form an alkyl radical and an iodine atom. Photofragment anisotropies demonstrate that the dissociation is fast relative to molecular rotation, ${ }^{1-4}$ and the complete absence of structure in the optical absorption spectra strongly suggests that dissociation is also faster than vibrational motion along any Franck-Condon active bound coordinate. The observation that the I product is formed in both its ground and excited states indicates that a curve crossing occurs between the ${ }^{3} Q_{0}$ surface, which carries most of the oscillator strength, and other surfaces, predominantly the ${ }^{1} Q_{1}$, and much experimental and theoretical study has gone into attempting to understand the dynamics of this curve-crossing process. ${ }^{2-12}$ The most extensive work has focused on the smallest member of the series $\left(\mathrm{R}=\mathrm{CH}_{3}\right)^{1,2,4,6,7,10-15} \mathrm{Methyl}$ iodide is an attractive subject for photodissociation studies because it appears to behave dynamically much like a diatomic or at least a linear triato-

\footnotetext{
a) Author to whom correspondence should be addressed.
}

mic molecule, ${ }^{7,12,13,16}$ while presenting a somewhat simpler set of potential surfaces (e.g., no nearby bound states) than most true diatomic or linear molecules (e.g., $I_{2}$ ).

More recently, several studies have compared a series of alkyl iodides in order to examine the effects of size and structure of the $\mathbf{R}$ group on the dissociation dynamics. The experimental quantities of particular interest in these studies have been the $I / I^{*}$ branching ratios and the energy partitioning in the photofragments. In general, it is observed that with increasing alkyl group size an increasingly large fraction of the I product is found in its electronic ground state, indicating that passage from the ${ }^{3} Q_{0}$ to the ${ }^{1} Q_{1}$ surface has occurred. ${ }^{3,5,8,9}$ A simple Landau-Zener model has been shown to explain semiquantitatively this increase in surface crossing probability with increasing alkyl group mass. ${ }^{3,8}$ In addition, as the size of the alkyl group is increased, and as the $\mathrm{R}$ group is changed from primary to secondary to tertiary, an increasing fraction of the total available energy appears in internal (rotational and vibrational) excitation of the alkyl fragment. ${ }^{3,9}$ While these variations in energy partitioning can be described empirically in terms of the "rigid" and "soft" radical models for the dissociation dynamics, ${ }^{17}$ they remain essentially unexplained. Furthermore, the experiments performed to date on the higher alkyl iodides do not distinguish the nature of the internal modes that are excited, 
although extensions of the mode-specific techniques which have already been applied to methyl iodide $e^{2,11,15,18-20}$ should be possible.

Resonance Raman spectroscopy has long been recognized as a powerful method for probing short-lived excited electronic states. The traditional sum-over-states picture of Raman scattering led early workers to focus on the relationship between resonance Raman intensities and the static excited-state potential surface(s) ${ }^{21-24}$ While knowledge of the potential surfaces implies knowledge of the dynamics of nuclear motion following photoexcitation (at least in the gas phase), it was the work of Lee and Heller, who interpreted the expression for the Raman amplitude in a time-dependent form ${ }^{25}$ that first focused attention on the utility of resonance Raman intensities for probing vibrational dynamics in reactive excited states. Early applications of resonance Raman to dissociative molecules involved diatomics ${ }^{26,27}$ (where the nature of the reaction coordinate is not in question) and polyenes in which the "dissociative" coordinate is a torsional mode. ${ }^{28-30}$ The method was first applied to methyl iodide by Kinsey and co-workers in the mid-1980s. ${ }^{18,19}$ Their data were at least qualitatively consistent with a picture in which the initial dynamics involves nearly pure stretching of the $\mathrm{C}-\mathrm{I}$ bond coupled to flattening of the $\mathrm{CH}_{3}$ dihedral angle at longer times, ${ }^{16}$ although efforts to simulate the spectra quantitatively have not been fully satisfactory, and the excitation profile data needed to verify the enhancement mechanism have not yet been published. More recently, the polarization properties of the emission have also been exploited to probe the dynamics of the curve-crossing process, ${ }^{10}$ and spectra have been obtained in several solvents in order to explore environmental effects on the dynamics. ${ }^{20}$

In the present paper, we take advantage of the modespecific information contained in resonance Raman intensities to examine the short-time dynamics of nuclear motion following $A$-state excitation of ethyl, isopropyl, and tert-butyl iodides. By measuring both relative and absolute scattering cross sections over a wide range of excitation frequencies both within and above the $A$-state absorption, we are able to show that even with $\boldsymbol{A}$-state resonant excitation the Raman fundamentals obtain much of their intensity from preresonance with higher electronic states, and we account for this preresonance contribution in our modeling of the spectra. We then make use of the ground-state normal-mode descriptions to convert the parameters of our spectral modeling into chemically meaningful internal coordinate dynamics. This provides detailed, atomic level pictures of the short-time (subvibrational period) dynamics of dissociation in each of the three molecules.

The present experiments have been carried out in cyclohexane solution mainly for reasons of technical convenience. Based on our previous work on methyl iodide ${ }^{20}$ we anticipate that the very short-time dynamics will be similar in vapor and solution phases. It would of course be extremely interesting to observe any solvent dependence of the dissociation dynamics. Spectra of ethyl, $n$-propyl, isopropyl, and tert-butyl iodides have been obtained in the vapor phase by Valentini and co-workers, ${ }^{31(a)}$ and a detailed comparison of the vapor- and solution-phase spectra will be reported separately. $^{31(\mathrm{~b})}$

\section{EXPERIMENTAL METHODS}

Ethyl, perdeuterated ethyl, isopropyl, and tert-butyl iodides (all from Aldrich) were used as obtained or after being distilled once. The Raman spectra of distilled and undistilled material appeared identical. The solvent was spectroscopic grade cyclohexane (EM Science). Excitation wavelengths of 199.8, 204.2, 208.8, 217.8, 223.1, 228.7, and $274 \mathrm{~nm}$ were generated by stimulated anti-Stokes Raman shifting the second, third, and fourth $(266 \mathrm{~nm})$ harmonics of a Quantel YG581 $Q$-switched Nd:YAG laser (where YAG denotes yttrium aluminum garnet) operating at $20 \mathrm{~Hz}$. Wavelengths of $302.6,284.6,276.6,268.8,270.3,278.3,258.2$, and $282.0 \mathrm{~nm}$ were generated from the second harmonic of a Nd:YAG pumped dye laser (Quantel TDL-50) or by stimulated antiStokes Raman shifting of the doubled dye laser. Figure 1 shows the relationship of these excitation wavelengths to the absorption spectra. Samples of approximately $75 \mathrm{ml}$ of 0.04 $M$ to $0.4 M$ alkyl iodide in cyclohexane solution were flowed

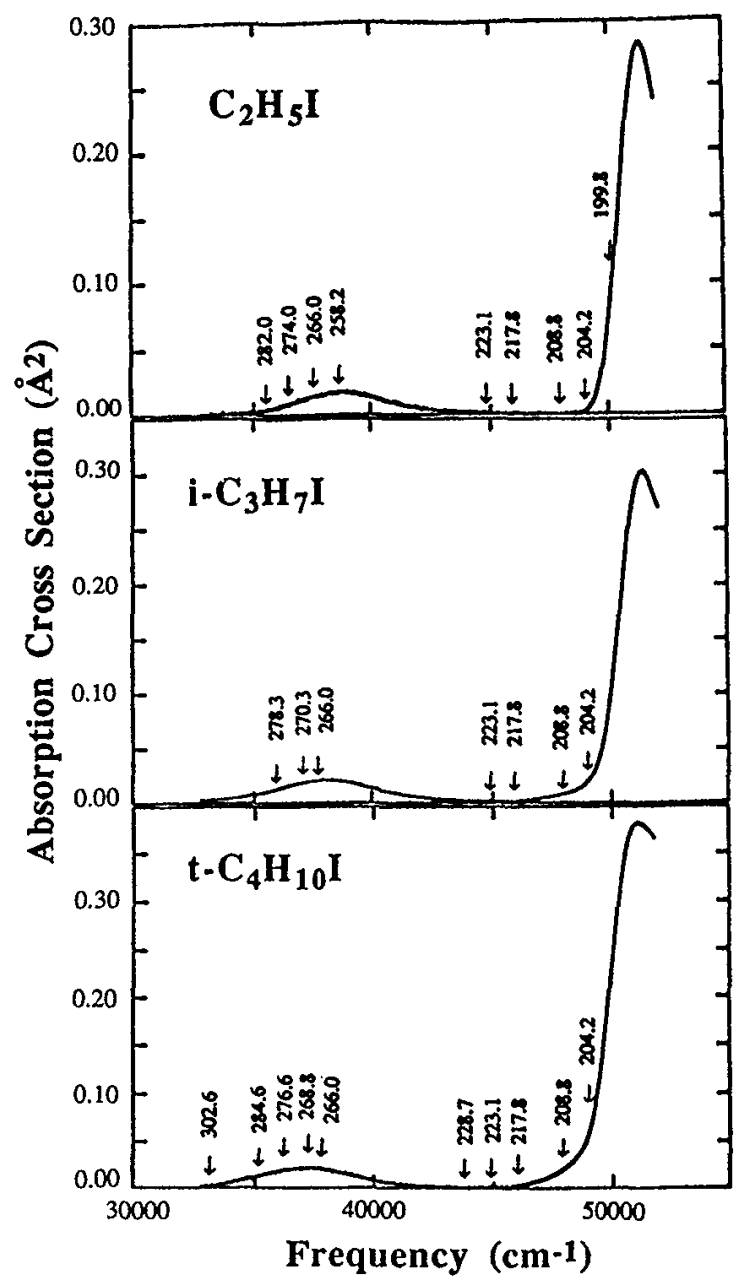

FIG. 1. Absorption spectra of ethyl iodide, isopropyl iodide, and tert-butyl iodide in cyclohexane solution. The excitation wavelengths (in $\mathrm{nm}$ ) at which resonance Raman spectra were obtained are indicated. The $A$-band absorption is the weak band between 30000 and $45000 \mathrm{~cm}^{-1}$, while the $B$ band absorption is the stronger band between 45000 and $55000 \mathrm{~cm}^{-1}$. 
through a dye jet to intersect the laser beam $(\sim 25$ $\mu \mathrm{J} /$ pulse), which was lightly focused to $1 \mathrm{~mm}$ diameter by a $0.5 \mathrm{~m}$ focal-length lens. The Raman scattering was detected in a backscattering geometry with the detected wave vector $\sim 135^{\circ}$ to the incident wave vector and the laser polarized perpendicular to the scattering plane. The Raman scattering was collected by an $f / 1.4$ ellipsoidal mirror (Spex) to eliminate distortion of the relative intensities by chromatic aberration, and imaged through a polarization scrambler (Karl Lambrecht) onto the entrance slit of a Spex $17020.75 \mathrm{~m}$ single spectrograph equipped with a 1200 groove $/ \mathrm{mm}$ ruled grating blazed at $300 \mathrm{~nm}$. A Princeton Instruments IRY$700 / \mathrm{N} / \mathrm{B}$ intensified diode array detector collected multichannel data. The signal was typically integrated for about $100 \mathrm{~s}$ on the detector before being read out, with $10-100$ of these scans added together and an equal number of dark scans subtracted to give the spectrum.

Depolarization ratios were measured by passing the incident laser through a calcite Glan-Taylor polarizer (Karl Lambrecht) to insure linear polarization, replacing the collection mirror with a fused silica lens system (Melles Griot), and placing a thin film uv polarization analyzer (Polarcoat 105-UV) in the Raman-scattered beam path just before the polarization scrambler. The detected polarization was rotated between parallel and perpendicular on successive accumulations, and the laser power for each scan was recorded by using a quartz plate to split off a piece of the incident beam into a solar blind photomultiplier (Hamamatsu R166) whose output was processed with a gated integrator/boxcar averager (Stanford Research Systems) interfaced to the computer. The measured depolarization ratios were corrected to account for the finite solid angle of collection as described by Dawson. ${ }^{32}$ In our experimental geometry this correction reduces the measured $\rho$ values by approximately $3 \%$.

In experiments that strive to obtain ground-state spectra using pulsed laser sources, it is vital that the laser power and focusing conditions be controlled such that photochemistry and/or ground-state depletion are not significant. The power dependence of the resonance Raman spectra and absolute cross sections was studied over a range from 5 to 250 $\mu \mathrm{J} /$ pulse. The spectra appeared unchanged up to 100 $\mu \mathrm{J} /$ pulse, while at $250 \mu \mathrm{J} /$ pulse the apparent absolute Raman cross sections were reduced by $\sim 15 \%$ from the lowenergy spectra. Thus, we take the $25 \mu \mathrm{J} /$ pulse as a safe level at which photoproduct accumulation and ground-state depletion are negligible. The Raman spectra also did not change over the $\sim 30 \mathrm{~min}$ data accumulation time for a particular sample. The resonance Raman spectra were calibrated in wave number using the known Raman frequencies of cyclohexane, various $\mathrm{H}_{2}$ Raman-shifted laser lines, and mercury emission lines. The entire optical system was corrected for the wavelength dependence and channel-to-channel variation of the detection efficiency by taking a spectrum of a calibrated intensity standard $\mathrm{D}_{2}$ lamp (Optronics model UV-40). The backscattering collection geometry used in these experiments minimizes distortions of the apparent relative intensities of different Raman lines caused by reabsorption of the scattered light; corrections for the remaining reabsorption error were performed as detailed previously. ${ }^{33}$
Relative peak areas were determined by fitting regions of the corrected spectra to a base line plus a sum of Lorentzians as discussed elsewhere. ${ }^{33}$

Absolute Raman cross sections for the alkyl iodides in cyclohexane solution were measured relative to the previously determined absolute Raman cross sections for cyclohexane summarized in Table $I$. The $802 \mathrm{~cm}^{-1}$ mode of cyclohexane was used as the internal standard for excitation wavelengths above $250 \mathrm{~nm},{ }^{34}$ and the sum of the cyclohexane $\mathrm{CH}$ stretch modes near $2900 \mathrm{~cm}^{-1}$ was used below 250 $\mathrm{nm} .^{35}$ The concentrations of the alkyl iodide/cyclohexane solutions were determined spectrophotometrically using a Cary 118 immediately before and after the Raman measurements. The maximum molar extinction coefficients in cyclohexane solution were found to be $445 M^{-1} \mathrm{~cm}^{-1}$ at 258.2 $\mathrm{nm}$ for ethyl iodide, $530 \mathrm{M}^{-1} \mathrm{~cm}^{-1}$ at $262.5 \mathrm{~nm}$ for isopropyl iodide, and $575 M^{-1} \mathrm{~cm}^{-1}$ at $268.8 \mathrm{~nm}$ for tert-butyl iodide, in good agreement with the values reported by $\mathrm{Ki}$ mura and Nagakura. ${ }^{36}$ Absorption spectra showed concentration changes of $5 \%$ or less during the experiment due to evaporation and photodecomposition. Average concentrations determined from the initial and final absorption spectra were used in the absolute cross-section calculations.

Since the alkyl iodides are relatively weak resonance $\mathrm{Ra}$ man scatterers, fairly high concentrations were needed to prevent the cyclohexane scattering from swamping the alkyl iodide Raman signal. Aggregation has been shown to shift both the band shape and the position of the absorption maximum of the $A$-band transition in vapor-phase methyl iodide. ${ }^{37}$ Markel and Myers examined the absorption spectrum of methyl iodide in hexadecane solution over a range of concentrations using short path-length cells and found negligible changes from $0.0075 M$ to $0.43 M{ }^{20}$ They also observed no change in the Raman spectra of methyl iodide over the concentration range $0.05 M-0.45 M$. We have also examined the Raman spectra of the alkyl iodides studied here over a

TABLE I. Internal standard cyclohexane absolute Raman cross sections.

\begin{tabular}{ccc}
\hline & \multicolumn{2}{c}{ Absolute cross section $\left(\AA^{2} /\right.$ molecule $)$} \\
\cline { 2 - 3 } $\begin{array}{c}\text { Wavelength } \\
\text { (nm) }\end{array}$ & $\begin{array}{c}\text { CH stretches } \\
\sim 2900 \mathrm{~cm}^{-1}\end{array}$ & $802 \mathrm{~cm}^{-1}$ band \\
\hline 199.8 & $2.37 \times 10^{-9}$ & $\cdots$ \\
204.2 & $1.98 \times 10^{-9}$ & $\ldots$ \\
208.8 & $1.62 \times 10^{-9}$ & $\ldots$ \\
217.8 & $1.12 \times 10^{-9}$ & $\ldots$ \\
223.1 & $9.31 \times 10^{-10}$ & $\ldots$ \\
228.7 & $7.57 \times 10^{-10}$ & $\cdots$ \\
258.2 & $\ldots$ & $2.14 \times 10^{-11}$ \\
266.0 & $\ldots$ & $1.82 \times 10^{-11}$ \\
268.8 & $\ldots$ & $1.72 \times 10^{-11}$ \\
270.3 & $\ldots$ & $1.66 \times 10^{-11}$ \\
274.0 & $\ldots$ & $1.55 \times 10^{-11}$ \\
276.6 & $\ldots$ & $1.47 \times 10^{-11}$ \\
278.3 & $\ldots$ & $1.42 \times 10^{-11}$ \\
282.0 & $\ldots$ & $1.32 \times 10^{-11}$ \\
284.6 & $\ldots$ & $1.27 \times 10^{-11}$ \\
302.6 & & $9.20 \times 10^{-12}$ \\
\hline \hline
\end{tabular}

Calculated from A-term parameters of Refs. 34 and 35. 
concentration range of $0.04 M-0.4 M$ and observed no changes in the spectra. Based on these observations, we are fairly confident that solute-solute interactions are not significant despite the relatively high solute concentrations used in our studies.

\section{COMPUTATIONAL METHODS}

Resonance Raman spectra are often analyzed under the assumption that the Raman enhancement arises solely from the electronic state(s) with which the excitation is resonant. In the present case, however, our preliminary results (see below) strongly indicated that preresonance enhancement from higher electronic states makes a significant contribution to the intensity even when exciting directly on resonance with the dissociative $A$ state, with the resulting resonance-preresonance interference leading to deenhancement of the fundamental Raman intensities as the excitation is tuned from the red edge of the $A$ band toward the absorption maximum. These "Raman antiresonance" effects have long been known experimentally and understood theoretically, ${ }^{38-44}$ and although the possible importance of preresonant contributions to the intensity is often ignored by workers wishing to interpret intensities in terms of the resonant-state dynamics, our wavelength-dependent data make it clear that neglect of such effects cannot be justified for the $A$-state alkyl iodides.

Accordingly, the absorption spectra and the $A$-state resonant absolute Raman intensities were modeled as follows. First, the preresonant contribution was accounted for by fitting the intensities in the region to the blue of the $A$-state absorption ( $230-200 \mathrm{~nm}$ ) to a single-state $A$-term frequency dependence, ${ }^{34,45}$

$$
\begin{aligned}
& \sigma_{R}\left(E_{L}\right)=E_{L} E_{S}^{3}\left\{A_{\mathrm{pre}}\right\}^{2}, \\
& \left\{A_{\mathrm{prc}}\right\}^{2}=K\left[\frac{E_{e}^{2}+E_{L}^{2}}{\left(E_{e}^{2}-E_{L}^{2}\right)^{2}}\right]^{2},
\end{aligned}
$$

where $E_{L}$ and $E_{s}$ are the laser and scattered photon energies in wave numbers, $E_{e}$ is the energy of the resonant electronic state, and $K$ is a coupling strength. $K$ and $E_{e}$ were varied to find the best fit to the preresonant data. Equation (1) provides a convenient fitting function with a realistic theoretical foundation, although in reality it is unlikely that a singlestate $A$-term dependence will be fully adequate to describe the frequency dependence of the intensities over a wide range of excitation frequencies. We considered the preresonance fit as a first approximation which was adjusted slightly (see below) in modeling the resonant data.

We then proceeded to model the $A$-state absorption spectrum and resonance Raman intensities via the time-dependent wave-packet approach described in detail elsewhere, ${ }^{46}$ modified to account for the preresonant contribution to the Raman amplitude. The absorption spectrum was computed from the usual expression,

$$
\begin{aligned}
\sigma_{A}\left(E_{L}\right)= & \frac{4 \pi e^{2} M^{2} E_{L}}{3 n \hbar^{2} c} \operatorname{Re} \int_{0}^{\infty} d t\langle 0 \mid 0(t)\rangle \\
& \times \exp \left[i\left(E_{L}+\epsilon_{0}\right) t / \hbar\right],
\end{aligned}
$$

where $M$ is the transition length which is considered to in- clude any local field factors, $E_{L}$ is the excitation energy, $n$ is the solvent refractive index, $\epsilon_{0}$ is the ground-state zero-point vibrational energy, and $|0(t)\rangle=\exp (-i H t / \hbar)|0\rangle$ is the initial multidimensional vibrational state propagated by the excited-state vibrational Hamiltonian. ${ }^{25}$ No thermal population of initial vibrational levels other than the ground state was considered, and due to the rapid damping of the overlaps caused by the wave-packet motion alone, no additional damping in the form of population or pure dephasing decay was employed.

For the Raman intensity calculations, we assumed that the resonant amplitude arises entirely from a single electronic state. In reality the $A$-band absorption consists of transitions to three different states, but magnetic circular dichroism data indicate that the ${ }^{3} Q_{0}$ state carries $70 \%-80 \%$ of the total oscillator strength. ${ }^{47}$ Since the resonance Raman intensities scale as the square of the oscillator strength, the weaker transitions will make a relatively small contribution to the intensities and have been neglected for simplicity, although their omission may lead to some error as discussed below. Even with the assumption of a single resonant state, however, we found it necessary to consider the interference between the preresonant and resonant amplitudes, and the nature of this interference depends on whether the resonant and preresonant amplitudes contribute to the same or to different tensor components of the Raman polarizability. (Note that the otherwise detailed treatment of Ref. 44 assumes throughout that only a single tensor component is involved.) For this purpose we treat the alkyl iodides as cylindrically symmetric molecules; this should be a reasonable approximation since the most important electronic transitions should be localized on the C-I moiety. With this assumption the Raman polarizability has only two different tensor components, $\alpha_{\gamma \gamma}$ and $\alpha_{\beta \beta}$, where $\gamma$ represents the symmetry axis and $\beta$ the doubly degenerate axes. The quantity we measure directly in our scattering geometry is then given by (see Refs. 48 and 49 for lucid summaries)

$$
\begin{aligned}
(d \sigma / d \Omega)_{\|+1}= & \frac{4 E_{S}^{3} E_{L}}{15(\hbar c)^{4}}\left[\left|\alpha_{\gamma \gamma}\right|^{2}+\frac{9}{4}\left|\alpha_{\beta \beta}\right|^{2}\right. \\
& \left.+\frac{1}{4}\left(\alpha_{\gamma \gamma}^{*} \alpha_{\beta \beta}+\alpha_{\beta \beta}^{*} \alpha_{\gamma \gamma}\right)\right] .
\end{aligned}
$$

The total Raman cross section, integrated over all directions and polarizations of the scattering, is given by

$$
\sigma_{T}\left(E_{L}, E_{s}\right)=\frac{8 \pi}{3}\left(\frac{1+2 \rho}{1+\rho}\right)(d \sigma / d \Omega)_{\|+1},
$$

where $\rho$ is the depolarization ratio, expressed in terms of the components of the Raman tensor by

$$
\rho=\frac{\left|\alpha_{\gamma \gamma}\right|^{2}+\left|\alpha_{\beta \beta}\right|^{2}-\left(\alpha_{\gamma \gamma}^{*} \alpha_{\beta \beta}+\alpha_{\beta \beta}^{*} \alpha_{\gamma \gamma}\right)}{3\left|\alpha_{\gamma \gamma}\right|^{2}+8\left|\alpha_{\beta \beta}\right|^{2}+2\left(\alpha_{\gamma \gamma}^{*} \alpha_{\beta \beta}+\alpha_{\beta \beta}^{*} \alpha_{\gamma \gamma}\right)} .
$$

In general, the depolarization ratio will be a function of excitation frequency due to the frequency dependence of $\alpha_{\gamma \gamma}$ and $\alpha_{\beta \beta}$.

We consider explicitly two extreme possibilities for the resonant-preresonant interference. The first is that both the resonant and the preresonant sources of intensity contribute solely to $\alpha_{\gamma \gamma}$. This would occur if the higher electronic states that give the dominant preresonant enhancement are all po- 
larized along the $\mathrm{C}-\mathrm{I}$ bond. In this case, the depolarization ratio should be $1 / 3$ independent of excitation frequency, and the total Raman cross section becomes

$$
\sigma_{T}\left(E_{L}, E_{S}\right)=\frac{10 \pi}{3}(d \sigma / d \Omega)_{\|+1},
$$

where

$$
(d \sigma / d \Omega)_{\|+1}=\frac{4 E_{S}^{3} E_{L}}{15(\hbar c)^{4}}\left|\alpha_{\mathrm{res}}+\alpha_{\mathrm{pre}}\right|^{2} .
$$

The resonant amplitude is calculated via the usual time-dependent expression, ${ }^{46}$

$\alpha_{\text {res }}=\frac{(e M)^{2}}{\hbar} \int_{0}^{\infty} d t\langle f \mid 0(t)\rangle \exp \left[i\left(E_{L}+\epsilon_{0}\right) t / \hbar\right]$,

while the preresonant amplitude is given in terms of the parameters of Eq. (1) as

$$
\left|\alpha_{\text {pre }}\right|^{2}=\left(9 \hbar^{4} c^{4} / 8 \pi\right)\left\{A_{\text {pre }}\right\}^{2} .
$$

(The question of the phase of $\alpha_{\text {pre }}$ is discussed below.) The other extreme occurs when the preresonant amplitude contributes only to $\alpha_{\beta \beta}$; that is, it comes entirely from transitions polarized perpendicular to the $\mathrm{C}-\mathrm{I}$ bond. In this case, multiplying $\alpha_{\beta \beta}$ through by a factor of $3 / 2$ for convenience, the differential cross section is given by

$$
\begin{aligned}
(d \sigma / d \Omega)_{\|+1}= & \frac{4 E_{S}^{3} E_{L}}{15(\hbar c)^{4}}\left[\left|\alpha_{\mathrm{res}}\right|^{2}+\left|\alpha_{\mathrm{pre}}\right|^{2}\right. \\
& \left.+\frac{1}{6}\left(\alpha_{\mathrm{res}}^{*} \alpha_{\mathrm{pre}}+\alpha_{\mathrm{res}} \alpha_{\mathrm{pre}}^{*}\right)\right],
\end{aligned}
$$

where $\alpha_{\text {res }}$ and $\alpha_{\text {pre }}$ are still obtained from Eqs. (8) and (9) above. In this case the depolarization ratio is, in general, a function of excitation wavelength and is given by

$\rho=\frac{\left|\alpha_{\mathrm{res}}\right|^{2}+\frac{4}{9}\left|\alpha_{\mathrm{pre}}\right|^{2}-\frac{2}{3}\left(\alpha_{\mathrm{pre}} \alpha_{\text {res }}^{*}+\alpha_{\mathrm{pre}}^{*} \alpha_{\mathrm{res}}\right)}{3\left|\alpha_{\mathrm{res}}\right|^{2}+\frac{32}{9}\left|\alpha_{\mathrm{pre}}\right|^{2}+\frac{4}{3}\left(\alpha_{\mathrm{pre}} \alpha_{\mathrm{res}}^{*}+\alpha_{\mathrm{pre}}^{*} \alpha_{\mathrm{res}}\right)}$.

In modeling the resonance-preresonance interference observed in these systems we have considered each of the above limits and have chosen the one most consistent with the data (see Sec. IV). The preresonant term was assumed to be negligible for all combination bands and overtones, which are observed to be very weak in the $230-200 \mathrm{~nm}$ region, as well as for some of the fundamentals which have little intensity at these wavelengths. The preresonant contribution to the amplitude was taken to be pure imaginary because the resonant term in Eq. (8) as written also approaches a pure imaginary quantity as the excitation is tuned away from resonance. The relative sign of the resonant and preresonant components is not necessarily known and depends on the relative direction of the geometry change along the coordinate of interest in the excited states responsible for the resonant and preresonant enhancements. We have assumed that for each coordinate the direction of the geometry change is the same in the resonant and preresonant states, so the preresonant term has the same sign as the preresonant limit of the resonant term. This seems well justified at least for the C-I stretch, which must undergo an increase in bond length in nearly all of its excited states. In the final calculations the magnitude of the preresonant term (but not its frequency dependence) was scaled by an overall constant ranging from
0.8 to 1.3 to best fit the fundamental intensities. This scaling is considered to be justified by the crudeness of the singlestate $A$-term approximation for describing the preresonance intensities.

The time-dependent overlaps, $\langle 0 \mid 0(t)\rangle$ and $\langle f \mid 0(t)\rangle$, were calculated as described previously ${ }^{46}$ with the assumption that the ground- and excited-state potential surfaces are separable harmonic oscillators having equal ground- and excited-state frequencies but different equilibrium geometries. This might appear to be a drastic oversimplification for a directly dissociative excited state. However, previous experience has shown that even a purely repulsive (e.g., exponential) potential can be quite well approximated for our purposes by a harmonic one, as long as the integration time in Eqs. (2) and (8) is made short enough that no recurrences appear and the displacement parameter $\Delta$ is large enough that all of the relevant overlaps approach zero by the time the integral is truncated. ${ }^{20}$ This is because, as long as no recurrences are allowed, both the absorption spectrum and the Raman intensities of the first few overtones depend mainly on the slope of the excited-state surface in the Franck-Condon region. ${ }^{30,50}$ While our model parameters cannot be expected to provide an adequate description of the excited-state potential surface far from the ground-state geometry, the multidimensional slope of the excited-state surface in the Franck-Condon region, and consequently the short-time dynamics of nuclear motion, are quite well determined by such a model.

The potential parameters used to fit Eqs. (2) and (8) are usually expressed in terms of computationally convenient dimensionless normal coordinates. In order to describe the dissociation dynamics in terms of actual atomic motions, we first express the motion of the center of the wave packet $|0(t)\rangle$ in dimensionless coordinates, and then convert to internal coordinates by making use of the normal-mode vectors. The position of the center of the wave packet at a time $t$ after excitation, for a system undergoing separable harmonic dynamics, is given in dimensionless normal coordinates by

$$
q_{\alpha}(t)=\Delta_{\alpha}\left(1-\cos \omega_{\alpha} t\right),
$$

where we set $q_{\alpha}=0$ for each mode $\alpha$ at the ground-state equilibrium geometry. The vibrational frequency $\omega_{\alpha}$, in units of fs ${ }^{-1}$, is given by $\bar{\omega}_{\alpha} / 5308.8$, where $\bar{\omega}_{\alpha}$ is in units of $\mathrm{cm}^{-1}$ and 5308.8 is the value of $\hbar$ in units of $\mathrm{cm}^{-1} \mathrm{fs}$. The dimensionless displacements $\left\{q_{\alpha}(t)\right\}$ are related to the internal coordinate displacements by ${ }^{5 t, 52}$

$$
s_{i}(t)=\left(\frac{h}{2 \pi c}\right)^{1 / 2} \sum_{\alpha} A_{\alpha i} \bar{\omega}_{\alpha}^{-1 / 2} q_{\alpha}(t),
$$

where $A_{\alpha i}$ is the normal-mode coefficient $\left(\partial s_{i} / \partial Q_{\alpha}\right), Q_{\alpha}$ is the ordinary (dimensioned) normal coordinate, and $s_{i}$ are the displacements of the internal coordinates (stretches, bends, torsions, and wags as defined by Wilson, Decius, and Cross $^{53}$ ) from their ground-state equilibrium values.

The normal-mode vectors for ethyl, isopropyl, and tertbutyl iodide were computed with a modification of the Snyder and Schachtschneider FG program described in detail elsewhere. ${ }^{51}$ The ground-state geometries and valence force fields were adapted from those previously published ${ }^{54-56}$ and have been adjusted slightly to better reproduce the experi- 
mental frequencies, which were also taken from Refs. 54-56. For ethyl iodide, an alternative calculation was performed using the experimental geometry recently obtained from analysis of the microwave spectra of isotopic derivatives. ${ }^{57}$ This geometry change (with no force-constant adjustments) gave typical frequency changes of $\sim 5 \mathrm{~cm}^{-1}$, with minor effects on the normal-mode coefficients.

\section{RESULTS}

\section{A. Absorption spectra}

Figure 1 shows the absorption spectra of ethyl, isopropyl, and tert-butyl iodides in cyclohexane solution and indicates the excitation frequencies used for the resonance Raman experiments. All of the alkyl iodides have very similar absorption spectra with an $A$-band maximum of moderate intensity near $260 \mathrm{~nm}$ and a much more intense $B$-band maximum near $196 \mathrm{~nm}$. The $A$-band absorption is composed of transitions to three directly dissociative electronic states $\left({ }^{3} Q_{0},{ }^{1} Q_{1}\right.$, and $\left.{ }^{3} Q_{1}\right)$, with the ${ }^{3} Q_{0}$ transition accounting for

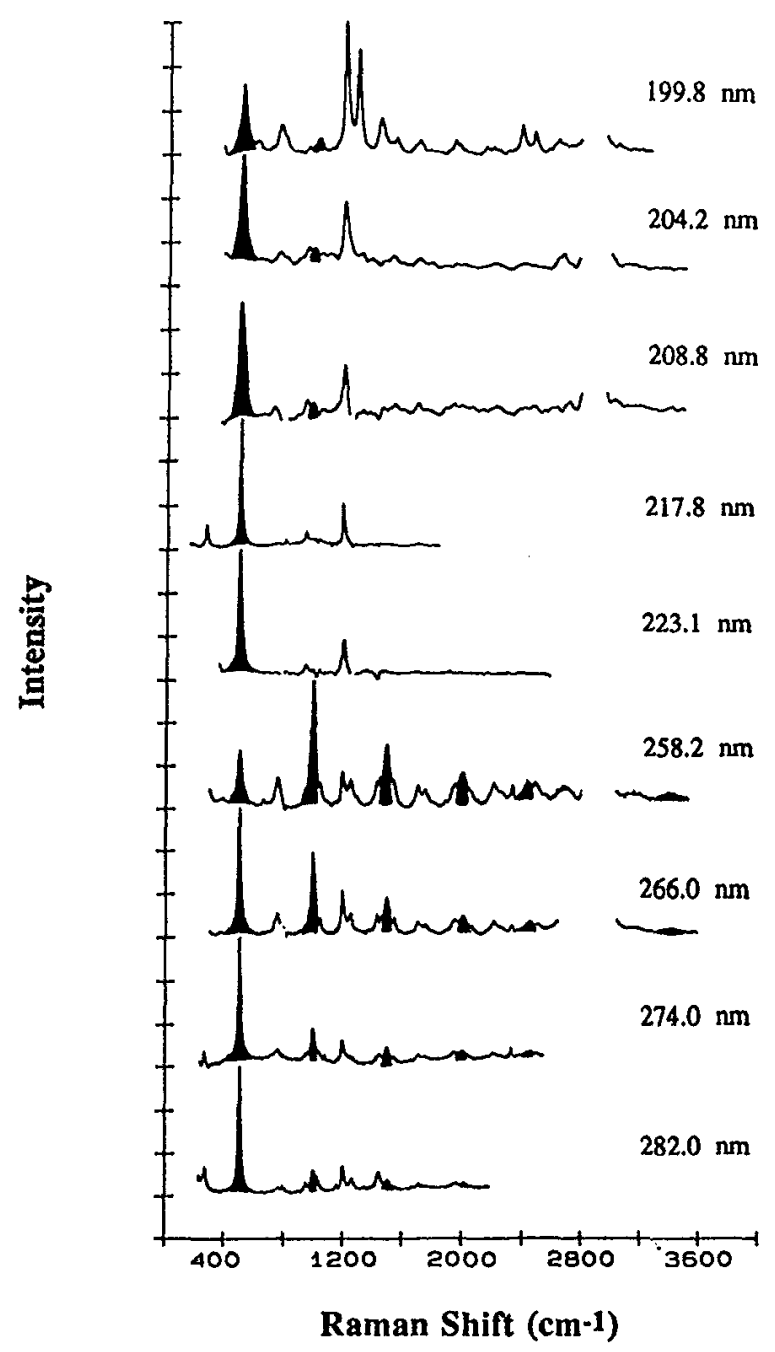

FIG. 2. Overview of the resonance Raman spectra of ethyl iodide in cyclohexane solution at the excitation wavelengths indicated. The $\mathrm{CH}$ stretch region is not shown due to a very large solvent subtraction. The spectra are intensity corrected and solvent subtracted. The shaded peaks are the nominal C-I stretch progression $\left(n v_{10}\right)$.
$70 \%-80 \%$ of the oscillator strength. ${ }^{47}$ It is only slightly affected by solvation and becomes progressively redshifted and more intense as the alkyl group becomes heavier and more branched. The $B$-band absorption is made up of transitions to a bound Rydberg state that predissociates to the lower, purely repulsive electronic states, ${ }^{58}$ and the gas-phase $B$-band absorption shows distinct vibronic structure in contrast to the structureless band observed in cyclohexane solution.

\section{B. Resonance Raman spectra}

Figures 2, 3, and 4 present overviews of the Raman spectra of ethyl, isopropyl, and tert-butyl iodides in cyclohexane, while Fig. 5 shows a more detailed view of the ethyl iodide spectrum at one excitation wavelength. The spectra are corrected for reabsorption and the wavelength dependence of the detection sensitivity as indicated in Sec. II, and are also solvent subtracted. Subtraction of the strong cyclohexane $\mathrm{CH}$ stretch bands around 2800 to $3100 \mathrm{~cm}^{-1}$ leaves that portion of the spectrum noisy and it is omitted. The nominal C-I stretch fundamental and overtones are shaded in the spectra of Figs. 2-5. The spectra of ethyl iodide at 258.2, 266, 274 , and $282 \mathrm{~nm}$ are resonant with the $A$-band absorption and show several progressions of overtones and combination bands. Most of the Raman intensity appears in the nominal

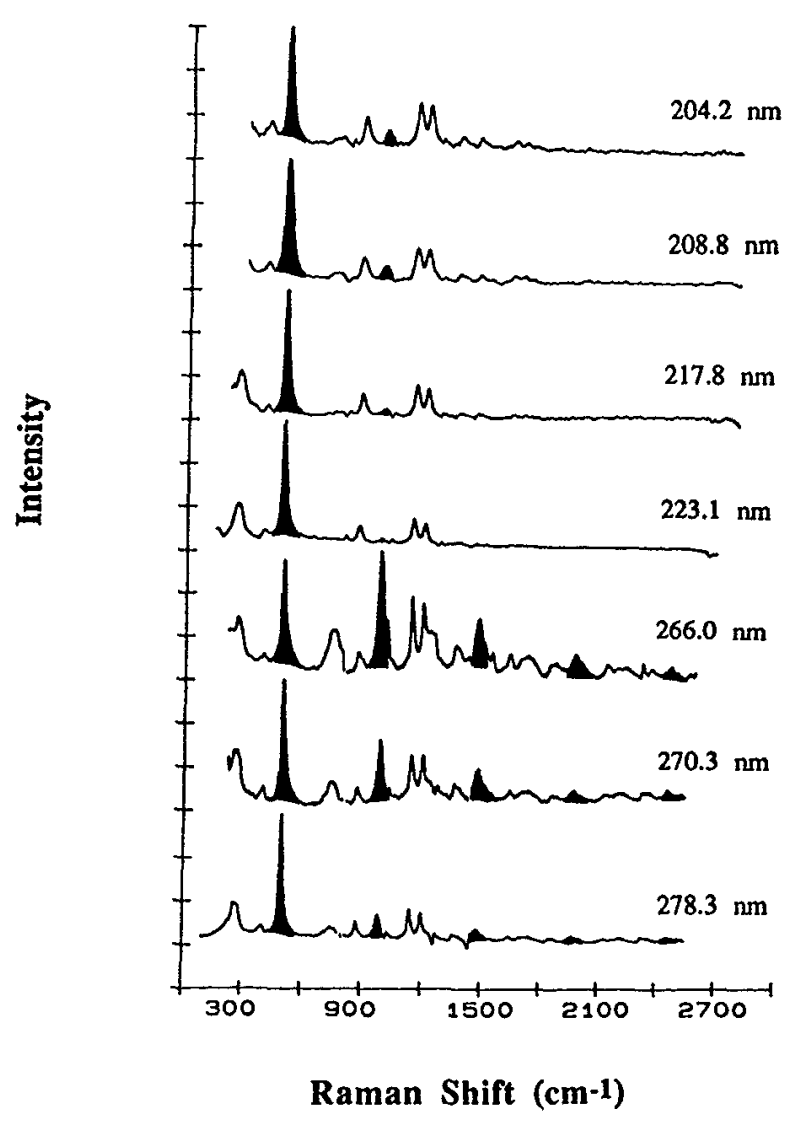

FIG. 3. Overview of the resonance Raman spectra of isopropyl iodide in cyclohexane solution at the excitation wavelengths shown. The spectra are intensity corrected and solvent subtracted. The $\mathrm{CH}$ stretch region is not shown due to a very large solvent subtraction. The shaded peaks are the nominal C-I stretch progression $\left(n v_{8}\right)$. 


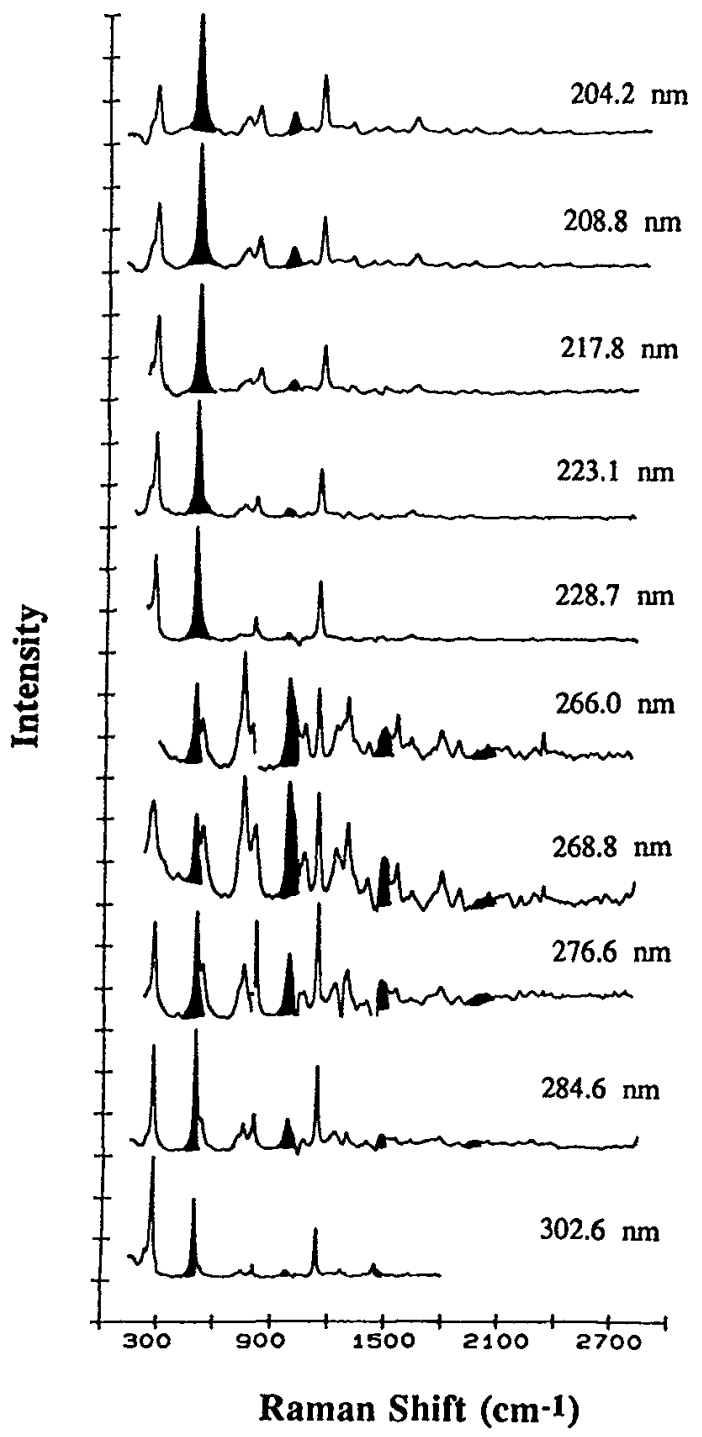

FIG. 4. Overview of the resonance Raman spectra of tert-butyl iodide in cyclohexane solution at the excitation wavelengths shown. The spectra are intensity corrected and solvent subtracted. The $\mathrm{CH}$ stretch region is not shown due to a very large solvent subtraction. The shaded peaks are the nominal C-I stretch progression $\left(n v_{7}\right)$.

C-I stretch fundamental and overtones $\left(n v_{10}\right)$. The next largest progression is the nominal $\mathrm{CCI}$ bend fundamental plus nominal C-I stretch combination bands $\left(v_{11}+n v_{10}\right)$. There are also three smaller combination bands made up of the $\mathrm{CH}_{3}$ rock, the $\mathrm{CH}_{2}$ wag, and the $\mathrm{CH}_{3}$ antisymmetric deformation fundamentals plus the nominal $\mathrm{C}-\mathrm{I}$ stretch $\left(v_{8}+n v_{10}, v_{7}+n v_{10}\right.$, and $v_{4}+n v_{10}$, respectively). The peak positions and intensities for the 258.2, 266, and $274 \mathrm{~nm}$ spectra are listed in Table II. The $199.8 \mathrm{~nm}$ spectrum is resonant with the $B$-band absorption and shows a distinctly different pattern of Raman intensities from the spectra taken resonant with the $A$-band absorption. The strongest feature in the $199.8 \mathrm{~nm}$ spectrum is the $\mathrm{CH}_{2}$ wag fundamental, $v_{7}$. The second strongest feature is a line at $1294 \mathrm{~cm}^{-1}$ which we have not been able to assign. It appears to be a combination band with the $v_{7}$ fundamental at $1202 \mathrm{~cm}^{-1} ;$ a $94 \mathrm{~cm}^{-1}$ interval appears both alone and as combination bands in the

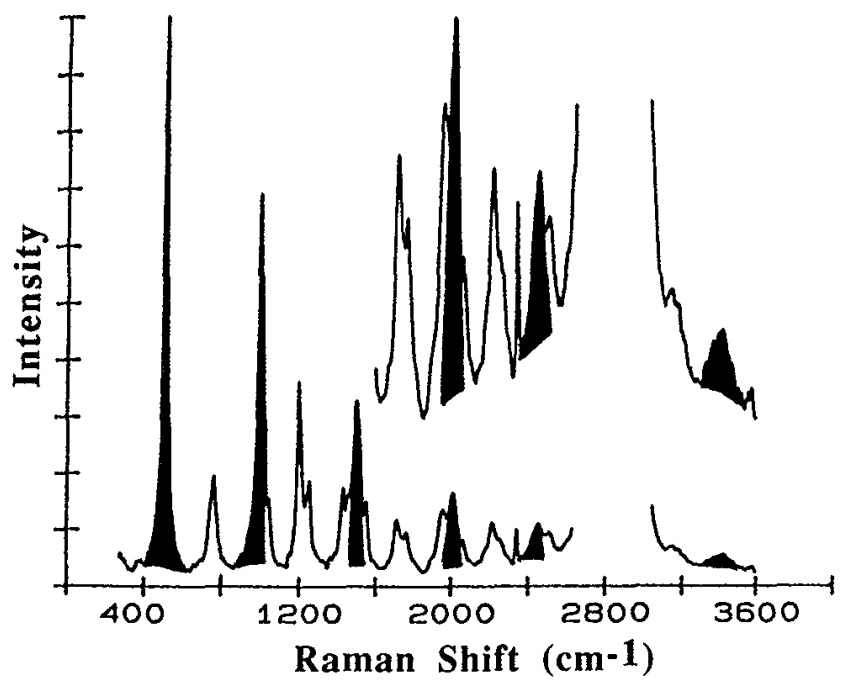

FIG. 5. Resonance Raman spectrum of ethyl iodide in cyclohexane solution taken with $266 \mathrm{~nm}$ excitation. The spectrum is intensity corrected and solvent subtracted. The region between 2700 and $3100 \mathrm{~cm}^{-1}$ is not shown due to noise resulting from solvent subtraction of the strong $\mathrm{CH}$ stretching bands of cyclohexane. The shaded peaks are the nominal C-I stretch progression $\left(n v_{10}\right)$.

$200 \mathrm{~nm}$ vapor-phase spectrum as well. ${ }^{59}$ The $\mathrm{CH}_{2}$ wag also shows overtones and a combination band with the nominal C-I stretch. The Raman spectra obtained at 204.2, 208.8, 217.8 , and $223.1 \mathrm{~nm}$ (between the $A$ - and $B$-band absorptions) are preresonant with the $B$-band Rydberg transition and show primarily fundamentals.

The Raman spectra of isopropyl iodide obtained with excitation wavelengths of $266,270.3$, and $278.3 \mathrm{~nm}$ show several combination bands and overtones. The largest progression in these $A$-band resonant spectra is the nominal C-I stretch fundamental and its overtones (shaded peaks in Fig. $3), n v_{8}$. The progression with the next largest intensity is the nominal $\mathrm{CCI}$ bending fundamental plus the nominal $\mathrm{C}-\mathrm{I}$ stretch combination bands $\left(v_{10}+n v_{8}\right)$. There are three other smaller combination band progressions: the CC stretch, the $\mathrm{CH}_{3}$ rock, and the $\mathrm{HCC}$ methine bend plus the nominal C-I stretch $\left(v_{7}+n v_{8}, v_{5}+n v_{8}\right.$, and $\left.v_{4}+n v_{8}\right)$. The peak positions and intensities for the 266, 270.3, and $278.3 \mathrm{~nm}$ Raman spectra are given in Table III. Spectra taken with excitation wavelengths of 204.2, 208.8, 217.8, and $223.1 \mathrm{~nm}$ (between the $A$-and $B$-band absorptions) are preresonant to the $B$-band Rydberg transition and therefore exhibit most of their intensity in fundamentals. As the excitation wavelength approaches the $B$-band absorption, the $v_{7}, v_{5}$, and $v_{4}$ fundamentals become slightly more intense relative to the C-I stretch.

The Raman spectra of tert-butyl iodide in cyclohexane are shown in Fig. 4. The spectra taken with excitation at 266, $268.8,276.6,284.6$, and $302.6 \mathrm{~nm}$ are resonant with the $A$ band absorption and show four sizable progressions of overtones and combination bands. The largest progression in these spectra is the nominal C-I stretch fundamental and its overtones (shaded peaks in Fig. 4 ), $n v_{7}$. The $v_{8}+n v_{7}$ combination band series has almost as much intensity. Both $v_{7}$ 
TABLE II. Experimental and calculated resonance Raman intensities for ethyl iodide in cyclohexane solution. ${ }^{a}$

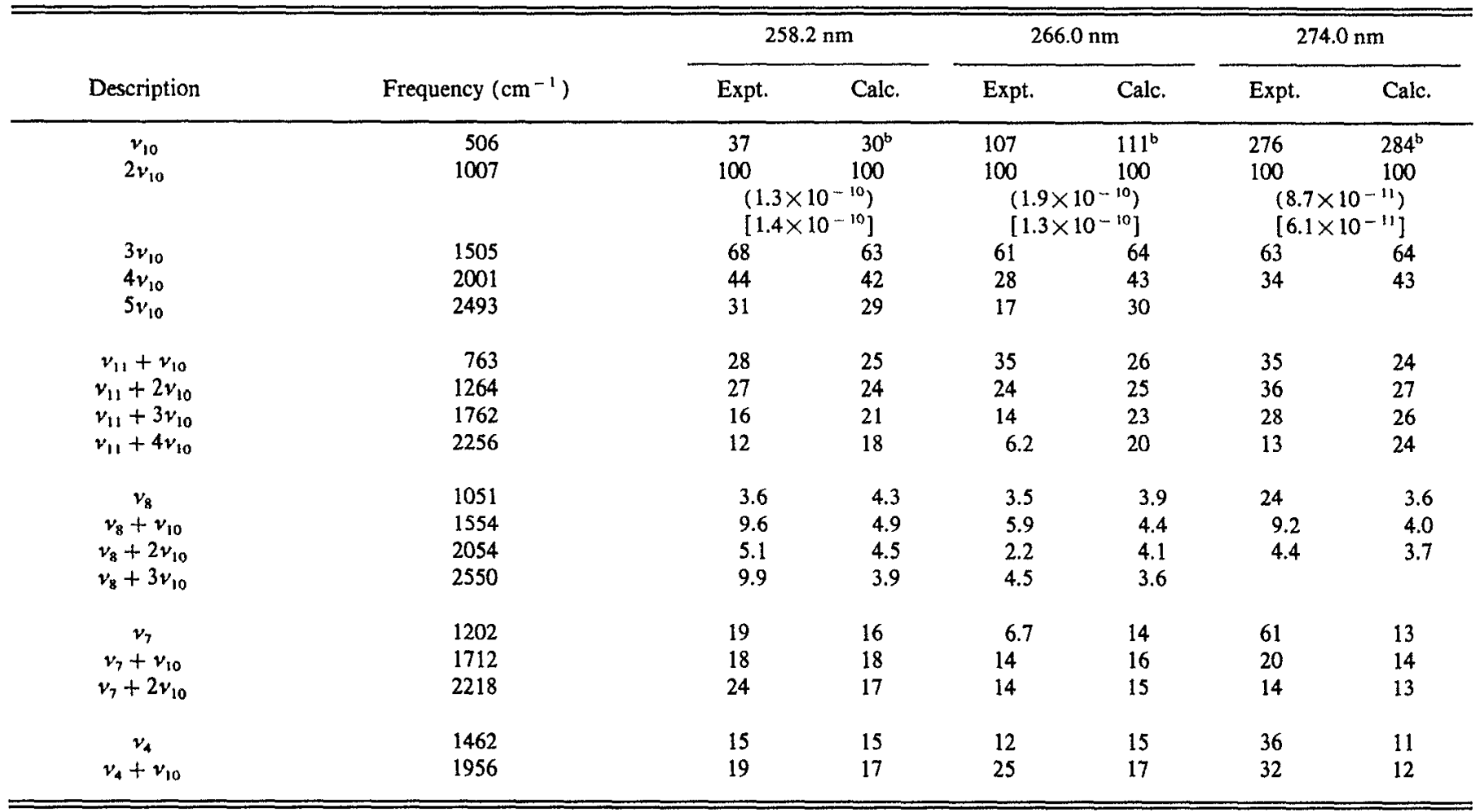

- Experimental total absolute cross section for $2 v_{10}$ in $\AA^{2} /$ molecule in parentheses; calculated value in square brackets.

'Preresonant contribution included in the intensity calculations (see text).

TABLE III. Experimental and calculated resonance Raman intensities for isopropyl iodide in cyclohexane solution."

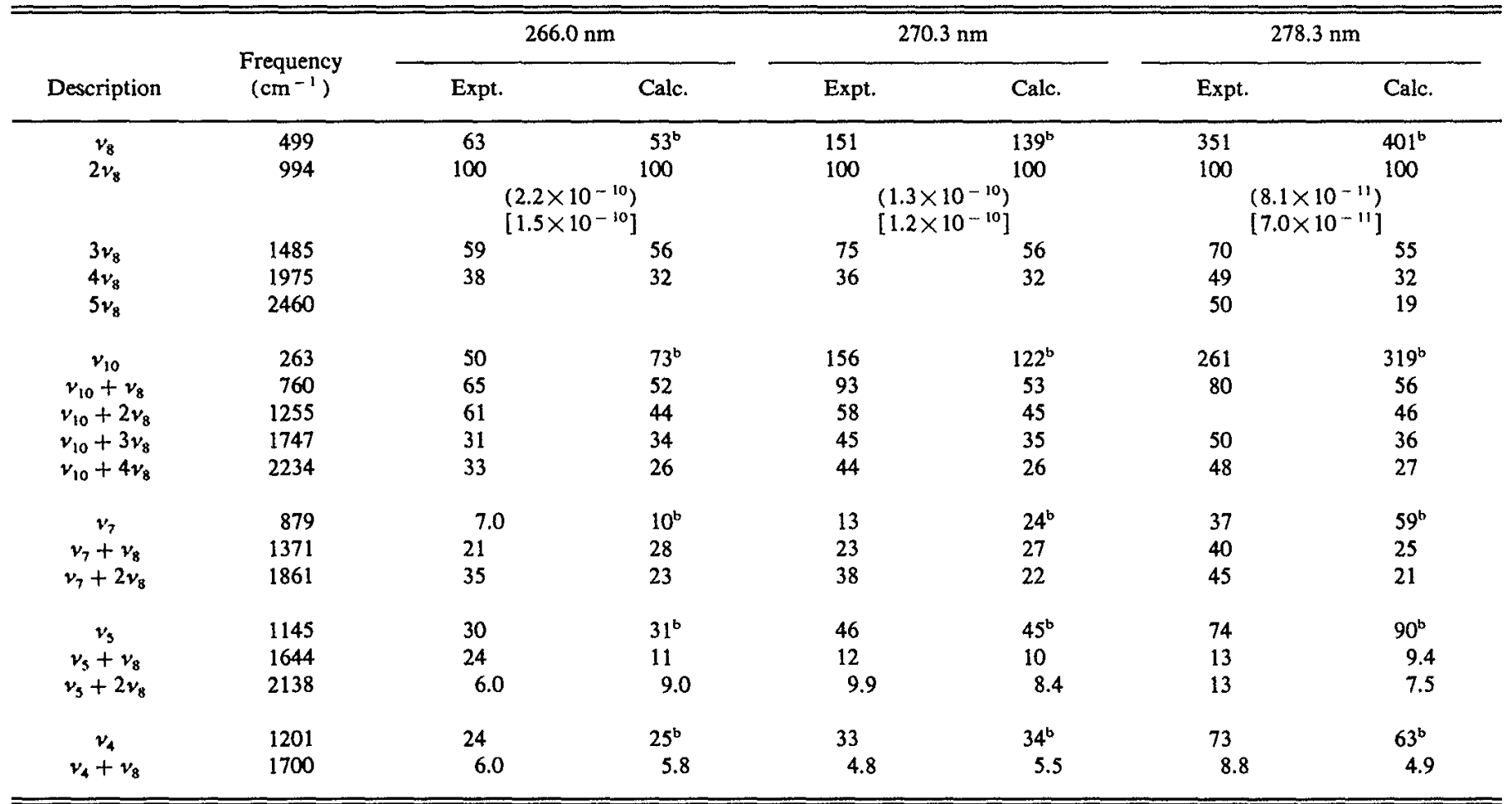

"Experimental total absolute cross section for $2 v_{8}$ in $\AA^{2} /$ molecule in parentheses; calculated value in square brackets.

'Preresonant contribution included in the intensity calculations (see text). 
TABLE IV. Experimental and calculated resonance Raman intensities for tert-butyl iodide in cyclohexane solution. ${ }^{2}$

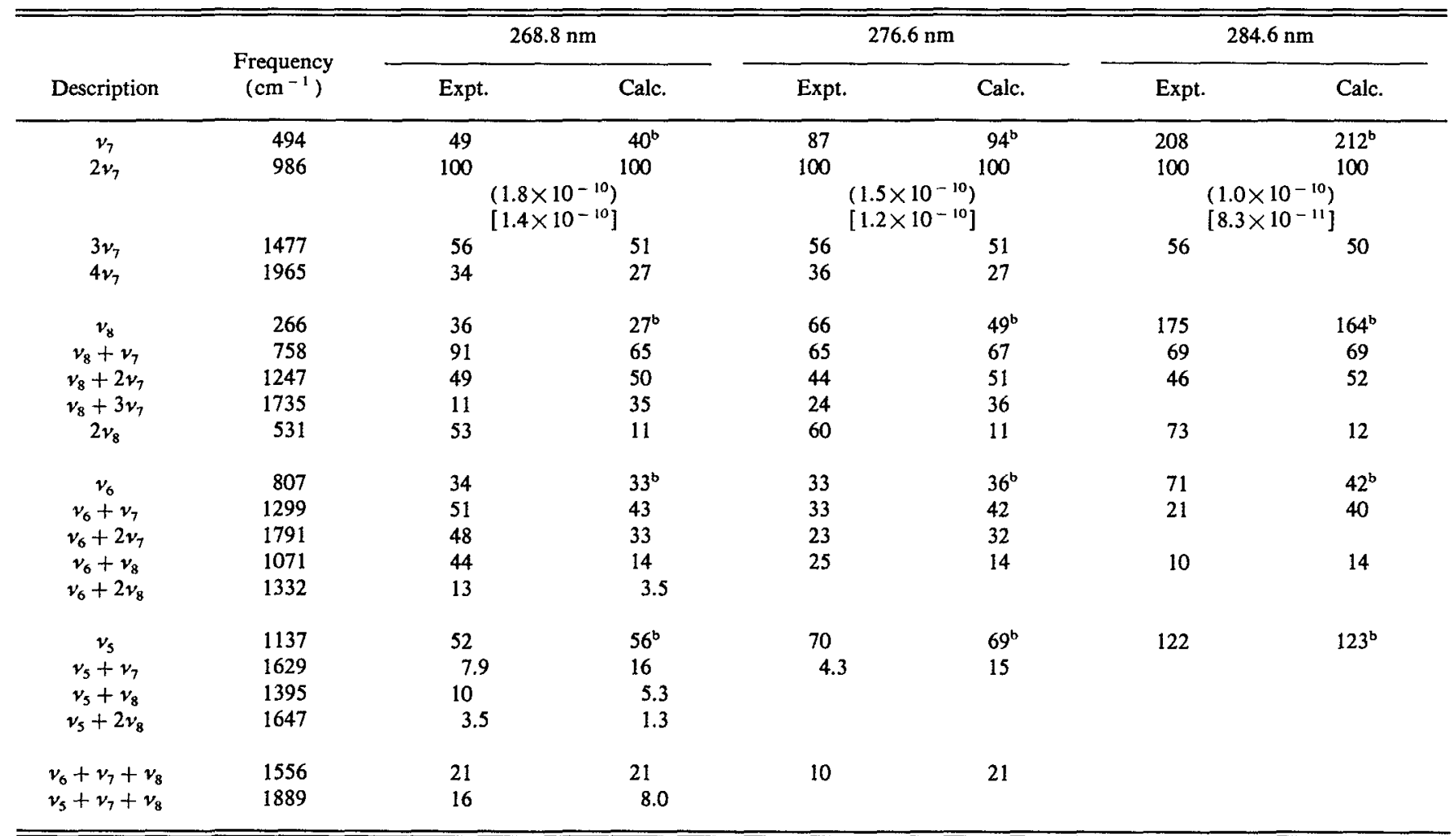

a Experimental total absolute cross section for $2 v_{7}$ in $\AA^{2} /$ molecule in parentheses; calculated value in brackets.

${ }^{b}$ Preresonant contribution included in the intensity calculations (see text).

and $v_{8}$ have large $\mathrm{CI}$ stretch internal coordinate contributions to their normal coordinates. There are two other smaller series of peaks associated with the nominal CC stretch and methyl rock fundamentals plus the $\mathrm{CI}$ stretch $\left(v_{6}+n v_{7}\right.$ and $\left.v_{5}+n v_{7}\right)$. The peak positions and intensities for the 268.8, 276.6, and $284.6 \mathrm{~nm}$ resonance Raman spectra are shown in Table IV. Spectra at 204.2, 208.8, 217.8, 223.1, and $228.7 \mathrm{~nm}$ (between the $A$ - and $B$-band absorptions) are preresonant to the $B$-band Rydberg transition and thus have most of their intensity in fundamentals.

The $A$-band resonance Raman spectra of the three alkyl iodides have common trends in their intensity patterns. The members of the nominal C-I stretch progression carry the most intensity. The second largest progression in all of the $A$ band spectra is the combination band series composed of one quantum of the mode near $260 \mathrm{~cm}^{-1}$ (predominantly CCI bending) and $n$ quanta of the $\mathrm{C}-\mathrm{I}$ stretch. The relative intensity in this progression increases as the alkyl group becomes heavier and more branched. This trend could be due either to participation of the CCI bending coordinate in the photodissociation dynamics or to an increasing amount of C-I stretch character in the mode around $260 \mathrm{~cm}^{-1}$ due to increased mixing of the internal coordinates in the groundstate normal modes. The same possible enhancement mechanisms exist for the other modes besides the nominal $\mathrm{CI}$ stretch which show intensity on resonance with the $A$ band. A quantitative analysis making use of the normal-mode de- scriptions is needed to determine the relative contributions of these two effects, and this is presented in succeeding sections.

Figure 6 presents the resonance Raman spectra of fully deuterated ethyl iodide at five excitation wavelengths spanning the $A$-band and $B$-band regions. Only $v_{10}, v_{11}$, and $v_{7}$ exhibit significant resonance intensity. Also, the unassigned line at $1294 \mathrm{~cm}^{-1}$ in the $199.8 \mathrm{~nm}$ spectrum of the parent molecule cannot be identified in perdeuterated ethyl iodide. Spectra of the deuterated species are of interest for two reasons. First, the $\mathrm{CH}$ stretching region of the alkyl iodide spectra is largely obscured by the very strong $\mathrm{CH}$ stretches of the cyclohexane solvent, making it difficult to evaluate the degree to which these modes participate in the photodissociation dynamics (although the absence of observable intensity in combination bands involving the $\mathrm{CH}$ stretches indicates that they are relatively inactive). The perdeuterated species shows relatively little intensity in CD stretching fundamentals, and most of the intensity that is observed appears to arise from preresonance with higher electronic states (note the intensity near $2200 \mathrm{~cm}^{-1}$ in the $204.2 \mathrm{~nm}$ spectrum). Second, the resonance Raman intensities in isotopically substituted species can be helpful in choosing among the many possible combinations of sign of the excited-state displacement parameters, as shown below. Table $V$ summarizes the relative intensities of the Raman lines of perdeuterated ethyl iodide observed with $A$-band resonant excitation. 


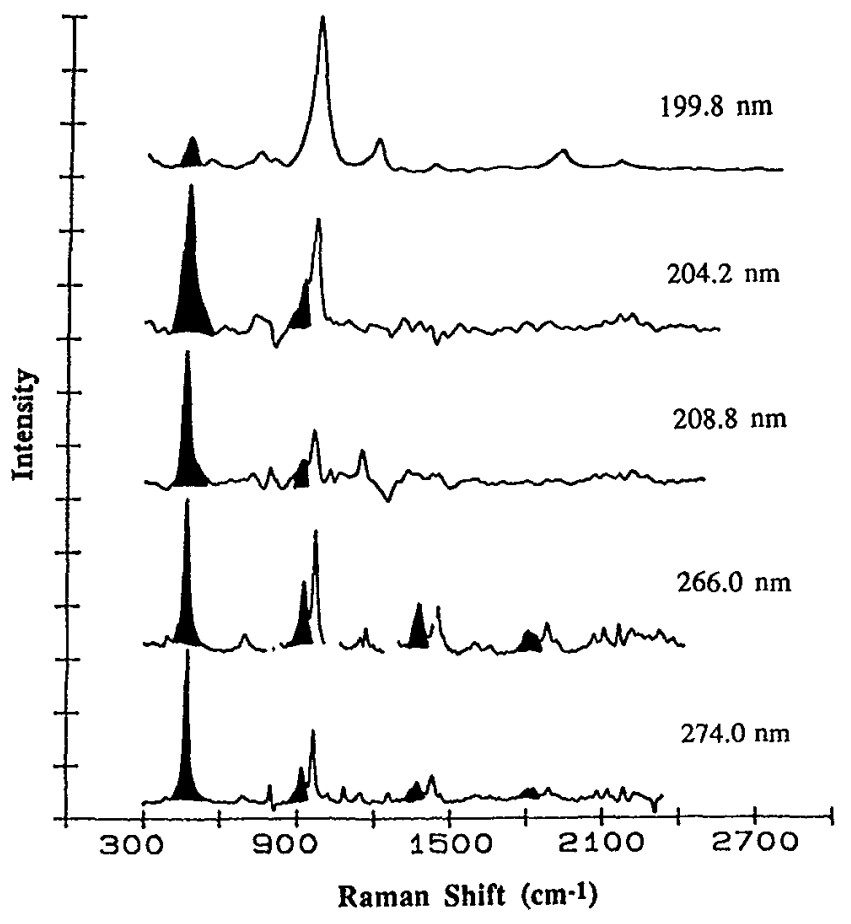

FIG. 6. Resonance Raman spectra of fully deuterated ethyl iodide in cyclohexane solution at the excitation wavelengths indicated. The spectra are intensity corrected and solvent subtracted. Small parts of the $266.0 \mathrm{~nm}$ spectrum are missing due to solvent subtraction artifacts. The shaded peaks are the nominal C-I stretch progression $\left(n v_{10}\right)$.

\section{Resonance Raman depolarization ratios and absolute Raman cross sections}

Depolarization ratios for the stronger Raman lines were measured for all three alkyl iodides at $266 \mathrm{~nm}$. Due to the difficulty of these experiments (the polarizer strongly attenuates the already weak scattered light) measurements were not attempted at other wavelengths. The depolarization ratio of the C-I stretching fundamental was found to be 0.37 ,

TABLE V. Experimental and calculated resonance Raman intensities for deuterated ethyl iodide in cyclohexane solution.

\begin{tabular}{ccccccc}
\hline & & \multicolumn{2}{c}{$266.0 \mathrm{~nm}$} & & \multicolumn{2}{c}{$274.0 \mathrm{~nm}$} \\
\cline { 5 - 6 } \cline { 5 - 6 } Description & $\begin{array}{c}\text { Frequency } \\
\left(\mathrm{cm}^{-1}\right)\end{array}$ & Expt. & Calc. & Expt. & Calc. \\
\hline$v_{10}$ & 463 & 176 & $163^{\mathrm{a}}$ & 389 & $404^{\mathrm{a}}$ \\
$2 v_{10}$ & 924 & 100 & 100 & 100 & 100 \\
$3 v_{10}$ & 1374 & 67 & 60 & 62 & 59 \\
$4 v_{10}$ & 1812 & 41 & 38 & 37 & 37 \\
& & & & & \\
$v_{11}+v_{10}$ & 692 & 29 & 34 & 22 & 36 \\
$v_{11}+2 v_{10}$ & 1148 & 26 & 31 & 44 & 32 \\
$v_{11}+3 v_{10}$ & 1601 & 27 & 26 & 40 & 27 \\
$v_{11}+4 v_{10}$ & 2046 & 18 & 21 & 40 & 22 \\
& & & & & \\
$v_{7}$ & 970 & 118 & $84^{\mathrm{a}}$ & 155 & $201^{\mathrm{a}}$ \\
$v_{7}+v_{10}$ & $1430^{\mathrm{b}}$ & $\mathrm{b}$ & 41 & $\mathrm{~b}$ & 37 \\
$v_{7}+2 v_{10}$ & 1882 & 39 & 36 & 33 & 32 \\
$v_{7}+3 v_{10}$ & 2327 & 30 & 30 & & \\
\hline \hline
\end{tabular}

- Preresonant contribution to calculated intensities included (see text).

${ }^{b}$ Large uncertainty in position and intensity due to subtraction of nearby $1444 \mathrm{~cm}^{-1}$ solvent band.
0.39 , and 0.34 (all \pm 0.03 ) for ethyl, isopropyl, and tertbutyl iodide, respectively. These are significantly but not greatly different from $1 / 3$, suggesting that the majority of the preresonant enhancement of the C-I stretching fundamental contributes to the same component of the Raman tensor as does the principal resonant contribution. The observation that the first few overtones of the C-I stretch have depolarization ratios similar to those of the fundamentals (near 0.39) supports this conclusion. Furthermore, in our modeling of the intensities we could not come close to reproducing the wavelength dependence of the fundamental cross sections by assuming orthogonally polarized resonant and preresonant amplitudes. As Eqs. (7) and (10) indicate, the interference terms are relatively much smaller when $\alpha_{\text {res }}$ and $\alpha_{\text {pre }}$ are orthogonally polarized, and the cross terms are then too small to generate the observed dispersion in the fundamental to overtone ratios regardless of whether an $A$-term or a $B$-term preresonance frequency dependence ${ }^{45}$ is assumed.

In all the analysis that follows, we assume that only a single element of the Raman tensor need be considered. This implies that the dominant source of preresonance enhancement is not the sharp $B$-band transition, which is known to be polarized perpendicular to the C-I bond, ${ }^{58}$ but rather other components of the broad quasicontinuum below 200 nm. ${ }^{60}$ The $A$ - and $B$-term preresonant fitting parameters further support this conclusion. For example, the $A$-term fit to the ethyl iodide $v_{10}$ fundamental intensity with excitation between 230 and $200 \mathrm{~nm}$ places the electronic state responsible for the preresonant enhancement at $58400 \mathrm{~cm}^{-1}$, well above the peak of the $B$-band absorption at about 51000 $\mathrm{cm}^{-1}$. A $B$-term fit puts the preresonant electronic state at $53500 \mathrm{~cm}^{-1}$ and the "virtual" state from which it borrows intensity at $125400 \mathrm{~cm}^{-1}$. While these parameters based on fits to the data over a limited frequency range cannot be taken too literally, they are at least consistent with the conclusion that electronic states other than the $B$ state are responsible for most of the preresonance enhancement.

The absolute Raman cross sections for the nominal C-I

TABLE VI. Nominal C-I stretch fundamental absolute Raman cross sections.

\begin{tabular}{cccc}
\hline \multirow{2}{*}{$\begin{array}{c}\text { Wavelength } \\
(\mathrm{nm})\end{array}$} & \multicolumn{3}{c}{ Absolute cross section $\left(\AA^{2} /\right.$ molecule $)$} \\
\cline { 2 - 4 } & Ethyl iodide & Isopropyl iodide & tert-butyl iodide \\
\hline 199.8 & $3.9 \times 10^{-8}$ & $\cdots$ & $\cdots$ \\
204.2 & $5.6 \times 10^{-9}$ & $1.4 \times 10^{-8}$ & $7.4 \times 10^{-8}$ \\
208.8 & $3.0 \times 10^{-9}$ & $6.6 \times 10^{-9}$ & $3.3 \times 10^{-8}$ \\
217.8 & $1.7 \times 10^{-9}$ & $3.6 \times 10^{-9}$ & $9.0 \times 10^{-9}$ \\
223.1 & $8.7 \times 10^{-10}$ & $2.3 \times 10^{-9}$ & $3.8 \times 10^{-9}$ \\
228.7 & $\ldots$ & $\cdots$ & $2.2 \times 10^{-9}$ \\
258.2 & $5.0 \times 10^{-11}$ & $\ldots$ & $\cdots$ \\
266.0 & $2.0 \times 10^{-10}$ & $1.4 \times 10^{-10}$ & $8.3 \times 10^{-11}$ \\
268.8 & $\ldots$ & $\cdots$ & $8.7 \times 10^{-11}$ \\
270.3 & $\ldots$ & $1.9 \times 10^{-10}$ & $\cdots$ \\
274.0 & $2.4 \times 10^{-10}$ & $\cdots$ & $\cdots$ \\
276.6 & $\ldots$ & $\cdots$ & $1.3 \times 10^{-10}$ \\
278.3 & $\ldots$ & $2.8 \times 10^{-10}$ & $\cdots$ \\
282.0 & $2.2 \times 10^{-10}$ & $\ldots$ & $\cdots$ \\
284.6 & $\ldots$ & $\ldots$ & $2.1 \times 10^{-10}$ \\
302.6 & $\ldots$ & $\cdots$ & $1.7 \times 10^{-10}$ \\
\hline \hline
\end{tabular}



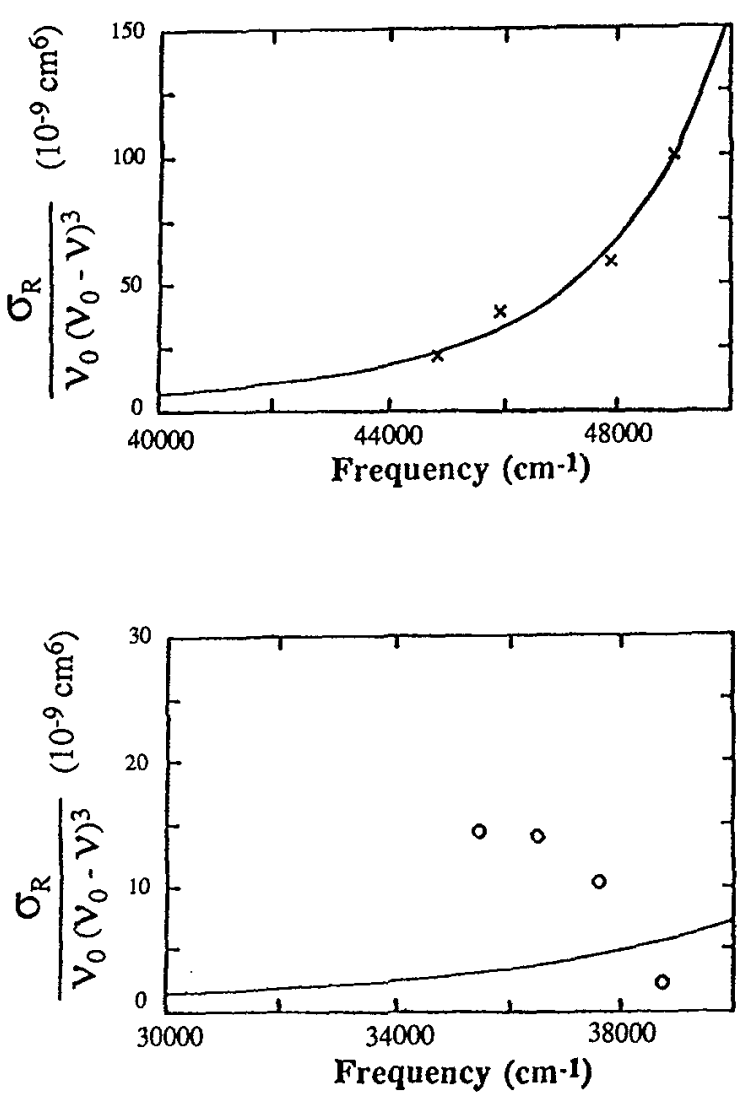

FIG. 7. Experimental absolute Raman cross sections of the $v_{10}$ fundamental of ethyl iodide taken with excitation wavelengths preresonant to the $B$-band absorption (X,top panel) and resonant with the $A$-band absorption $(\mathrm{O}$, bottom panel). The cross sections have been divided by the $v^{4}$ dependence with $v$ in $\mathrm{cm}^{-1}$. An Albrecht $A$-term fit to the preresonant absolute cross sections of the top panel is shown as the solid line, and its extrapolation into the $A$-band absorption region is shown in the bottom panel. The extrapolated preresonant contribution to the C-I stretch fundamental in the $A$-band region is not negligible compared with the experimental intensity.

stretching fundamental at the wavelengths indicated in Figs. 2-4 are given in Table VI. These total absolute cross sections have been calculated from the directly measured relative differential cross sections as discussed in Ref. 46 [see also Eq. (4) ] using depolarization ratios of 0.10 and 0.23 for the cyclohexane $802 \mathrm{~cm}^{-1}$ line and the sum of the $\mathrm{CH}$ stretches, respectively. ${ }^{35.52}$ As we were not able to measure accurate depolarization ratios for the weaker alkyl iodide bands, we have assumed $\rho=1 / 3$ throughout. Using $\rho=0.39$ rather than 0.333 would increase the conversion factor from differential to total cross section by $7 \%$.

The Raman cross sections for excitation between 200 and $230 \mathrm{~nm}$, preresonant with the $B$-state and higher Rydberg transitions, are much larger than those observed in the $A$-band region ( $250-300 \mathrm{~nm}$ ). Figure 7 shows the $A$-term fit to the nominal C-I stretch fundamental intensities at 200 $230 \mathrm{~nm}$ for ethyl iodide, as well as the extrapolation of this preresonance fit into the $A$-band absorption relative to the observed absolute Raman cross sections. The corresponding plots for isopropyl and tert-butyl iodides are similar. It is seen that the preresonant contribution to the intensity is comparable to the actual observed intensity with $A$-band resonant excitation, and clearly cannot be ignored. This may explain the discrepancy observed between theory and experiment for the intensity of the fundamental relative to the first overtone in previous work on methyl iodide..$^{16,20}$

\section{Absorption and resonance Raman spectral modeling}

The absorption spectra and $A$-band resonant absolute Raman cross sections for all three alkyl iodides as well as deuterated ethyl iodide have been modeled using Eqs. (2) and (6)-(9) as outlined in Sec. III. The calculations take into account the contributions of preresonance scattering to the fundamental intensities. The calculated and experimental relative intensities are compared in Tables II-V along with the absolute Raman cross section for the first overtone of the nominal C-I stretch. The spectra excited closer to the absorption maxima were given more weight than the ones near the red edge where the ${ }^{3} Q_{1}$ state starts to make a significant contribution to the absorption intensity. The parameters used in the modeling are given in Table VII. The calculated and experimental $A$-band absorption spectra, displayed in Fig. 8, show reasonable agreement considering that we are neglecting the minor contributions of the ${ }^{3} Q_{1}$ and ${ }^{1} Q_{1}$ states to the red and blue edges of the $A$-band absorption.

The calculated and experimental relative intensities for the 258.2, 266, and $274 \mathrm{~nm}$ ethyl iodide resonance Raman spectra are shown graphically in Fig. 9. At $258.2 \mathrm{~nm}$ the ${ }^{3} Q_{0}$ state contributes almost all of the absorption strength ac-

TABLE VII. Parameters of alkyl iodide simulations. ${ }^{\text {a }}$

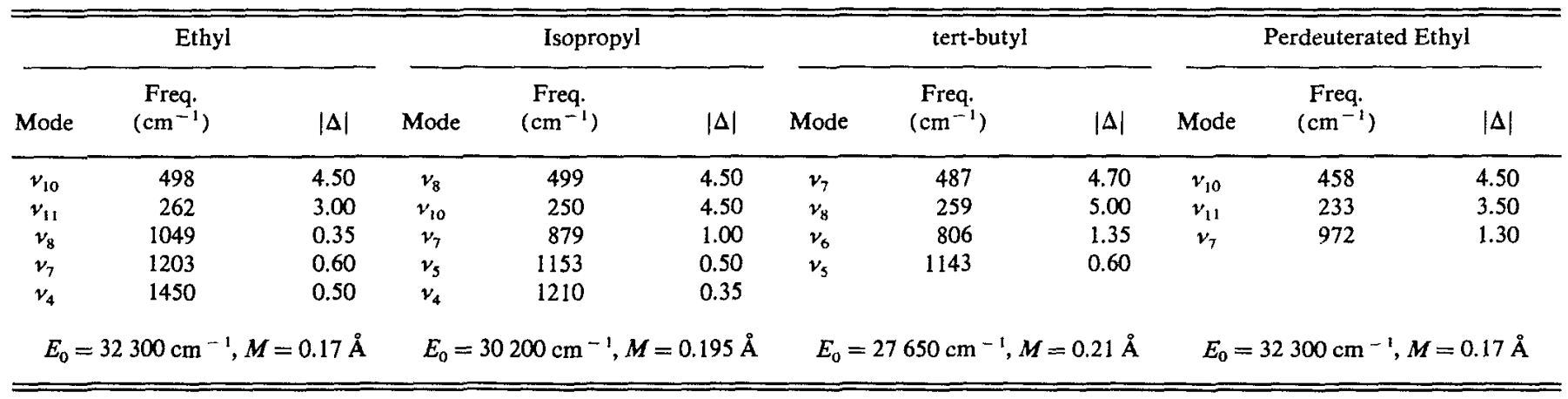

aSee Sec. III for a definition of the parameters. The "zero-zero energy," $E_{0}$, should not be taken literally, as these fitting parameters cannot be meaningfully extrapolated to an excited-state potential minimum (see the text). 


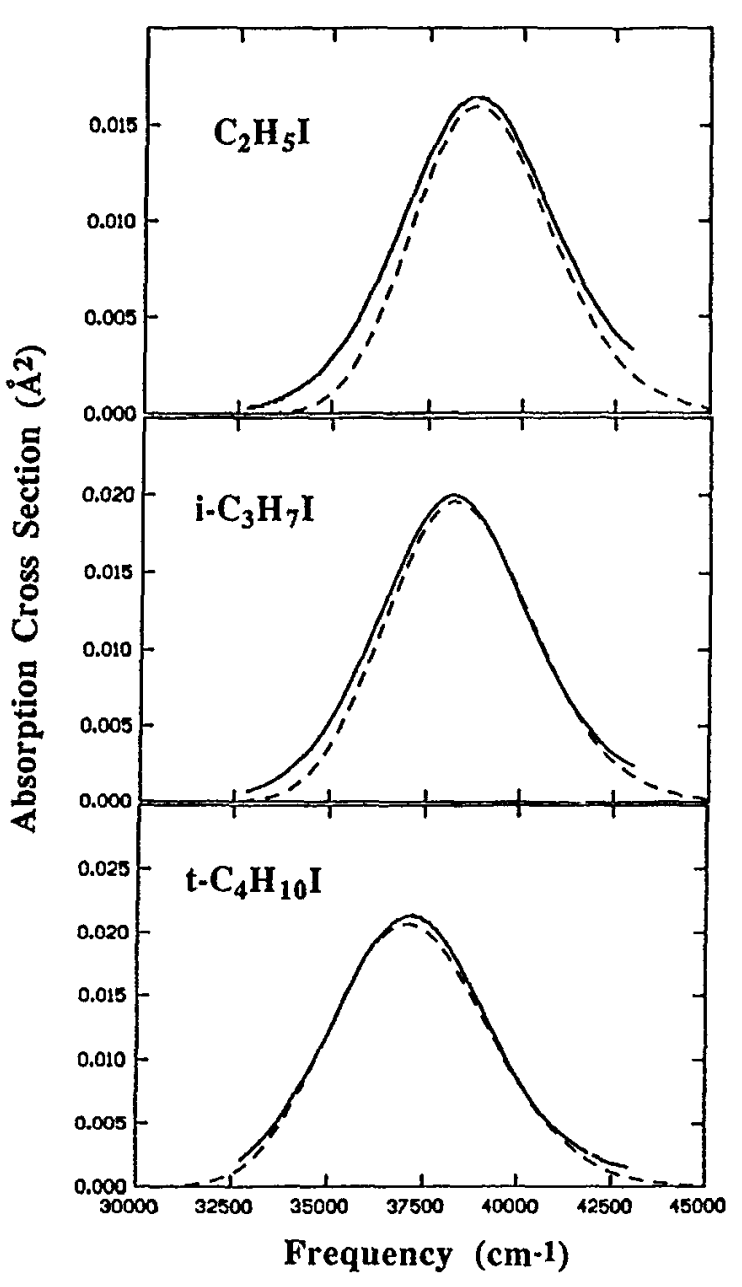

FIG. 8. $A$-band absorption cross sections of the alkyl iodides in cyclohexane solution, experimental (solid line) and calculated using the parameters of Table VII (dashed line).

cording to the magnetic circular dichroism (MCD) experiments, and the agreement between experimental and calculated intensities is excellent. The calculated and experimental intensities at 266 and $274 \mathrm{~nm}$ excitation are still in reasonable agreement, although there are some noticeable discrepancies, particularly at $274 \mathrm{~nm}$. This wavelength is near the peak of the weak ${ }^{3} Q_{1}$ absorption, and this state, not included in our calculations, may contribute to the experimental intensities. The calculated and experimental Raman intensities for isopropyl iodide at 266, 270.3, and $278.3 \mathrm{~nm}$ are listed in Table III and shown graphically in Fig. 10. The agreement between calculated and experimental intensities is generally good, though not as good as for ethyl iodide. No MCD experiments have been reported for isopropyl iodide so we do not know the exact composition of its $A$-band absorption. Isopropyl may have more of its absorption strength in the ${ }^{3} Q_{1}$ and ${ }^{1} Q_{1}$ states which may also be located at different wavelengths than in ethyl iodide, causing a somewhat larger perturbation on the resonance Raman spectra. The calculated and experimental Raman intensities for tert-butyl iodide at 268.8,276.6, and $284.6 \mathrm{~nm}$ are listed in Table IV and shown graphically in Fig. 11. Again the agreement between the calculated and experimental intensities is reasonably good, though not as good as for ethyl iodide. Here the ${ }^{3} Q_{1}$ and ${ }^{1} Q_{1}$ states do make a greater contribu-

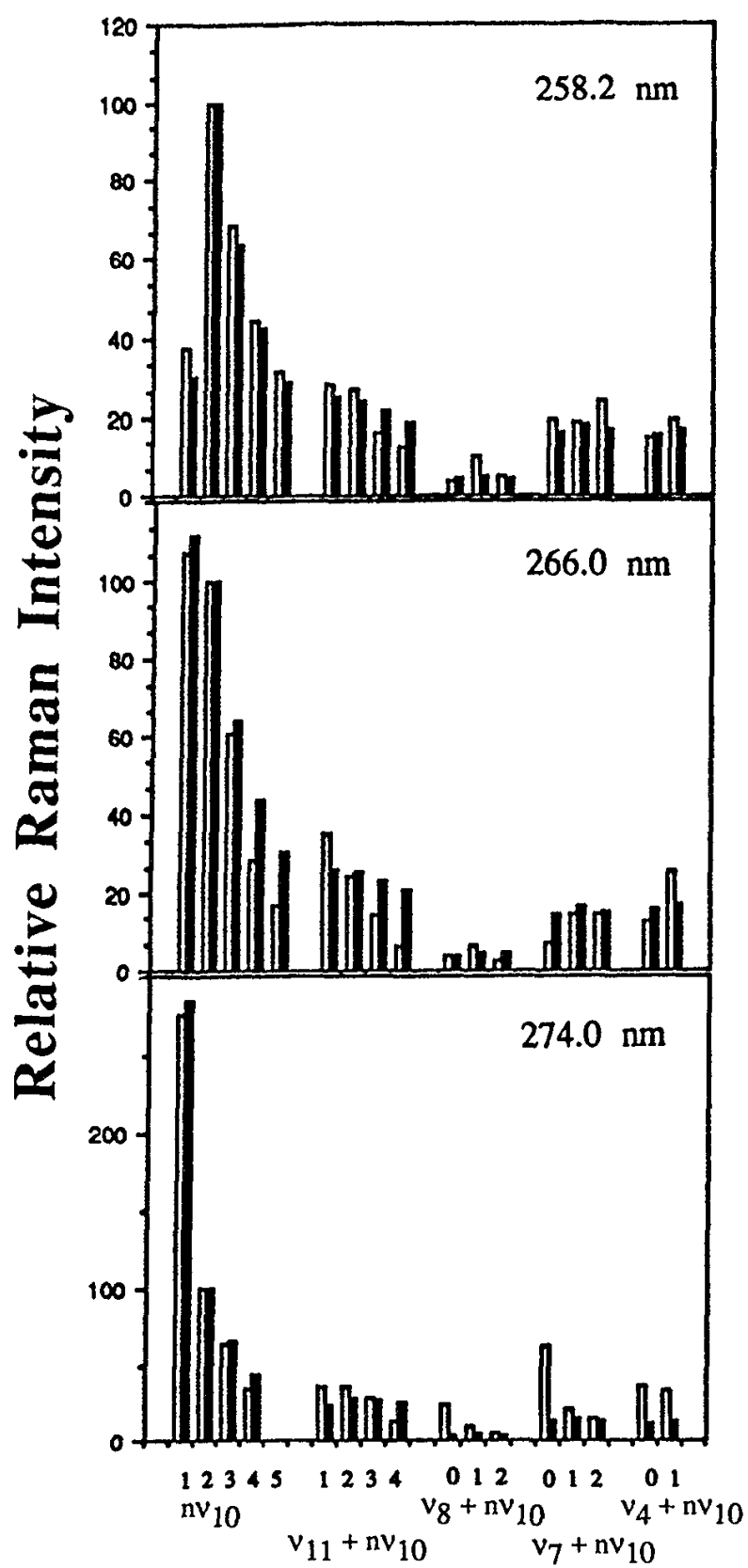

FIG. 9. Comparison of experimental (open bars) and calculated (solid bars) relative Raman intensities at three different excitation wavelengths in the $A$-band absorption of ethyl iodide in cyclohexane solution.

tion to the total $A$-band oscillator strength than in ethyl iodide; also, the ${ }^{3} Q_{1}$ state is located closer to the maximum of the absorption band in tert-butyl iodide than in ethyl iodide. $^{47}$

Table $\mathrm{V}$ compares the observed and calculated relative intensities for fully deuterated ethyl iodide at 266.0 and $274.0 \mathrm{~nm}$. We see very good agreement for most of the resonance Raman intensities. The $274.0 \mathrm{~nm}$ spectrum, which is likely to have a larger contribution from the weak ${ }^{3} Q_{1}$ absorption, does not show as good agreement between experiment and calculation as does the $266.0 \mathrm{~nm}$ spectrum. Our simulations reproduce well the larger $v_{10} / 2 v_{10}$ ratios at 274 and $266 \mathrm{~nm}$ in deuterated relative to normal ethyl iodide. 


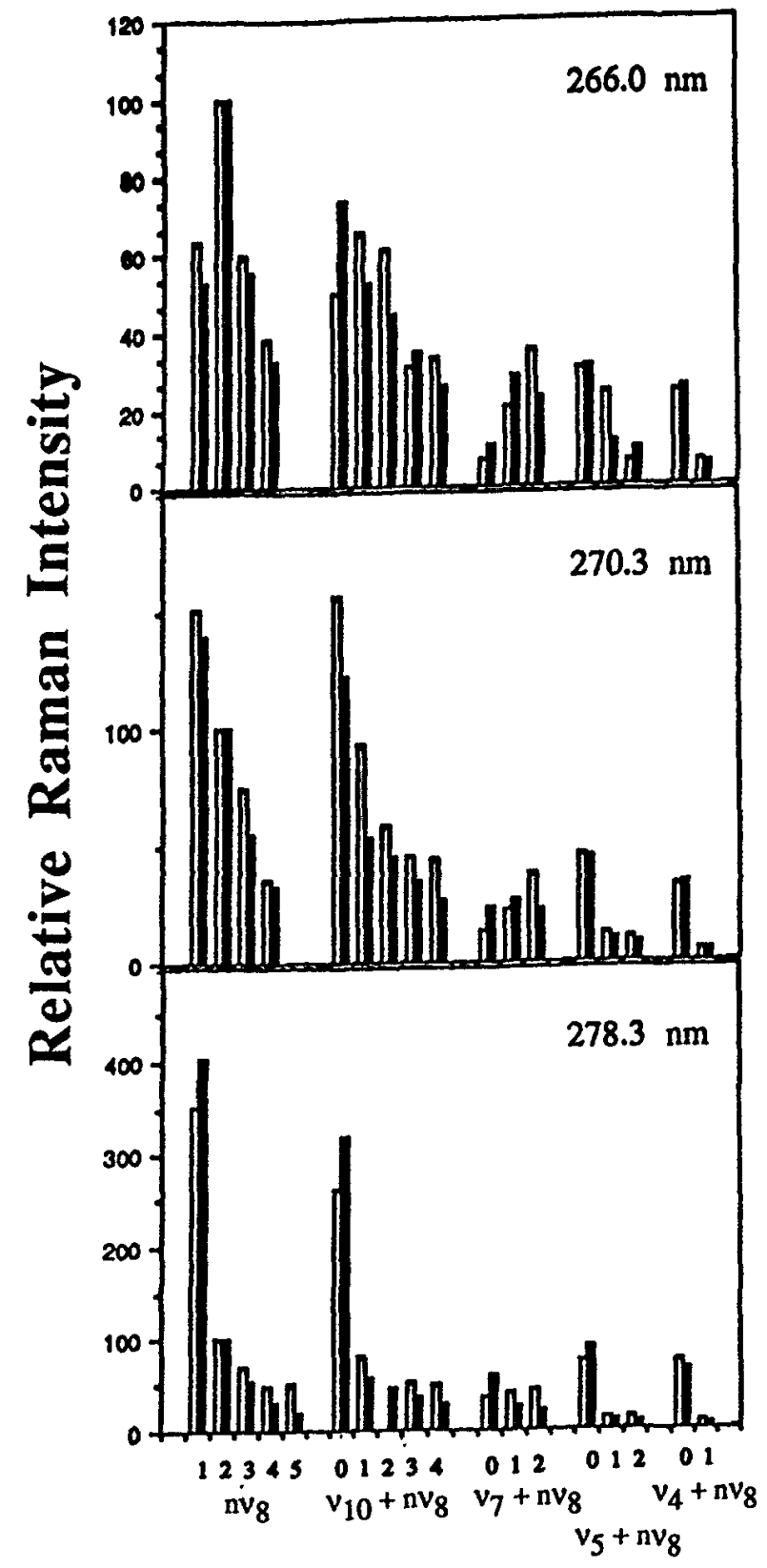

FIG. 10. Comparison of experimental (open bars) and calculated (solid bars) relative Raman intensities at three different excitation wavelengths in the $A$-band absorption of isopropyl iodide in cyclohexane solution.

This agreement may be largely artificial because we did not measure any absolute cross sections for the deuterated species, and accounted for the preresonant term by scaling the normal ethyl iodide $A$-term frequency dependence to best reproduce the deuterated data. However, the absorption spectrum is slightly narrower in the deuterated species ( $2250 \mathrm{~cm}^{-1}$ vs $2400 \mathrm{~cm}^{-1}$ in $h_{5}$-ethyl iodide) and this does affect the calculated frequency dependence of the fundamental intensity.

For comparison, we have carried out the same calculations for ethyl iodide omitting the preresonant contribution to the fundamental intensities. Figure 12 shows the experimental ratio of the fundamental to the first overtone of the nominal C-I stretch compared to the ratios calculated with and without the preresonant contribution. This figure dem-

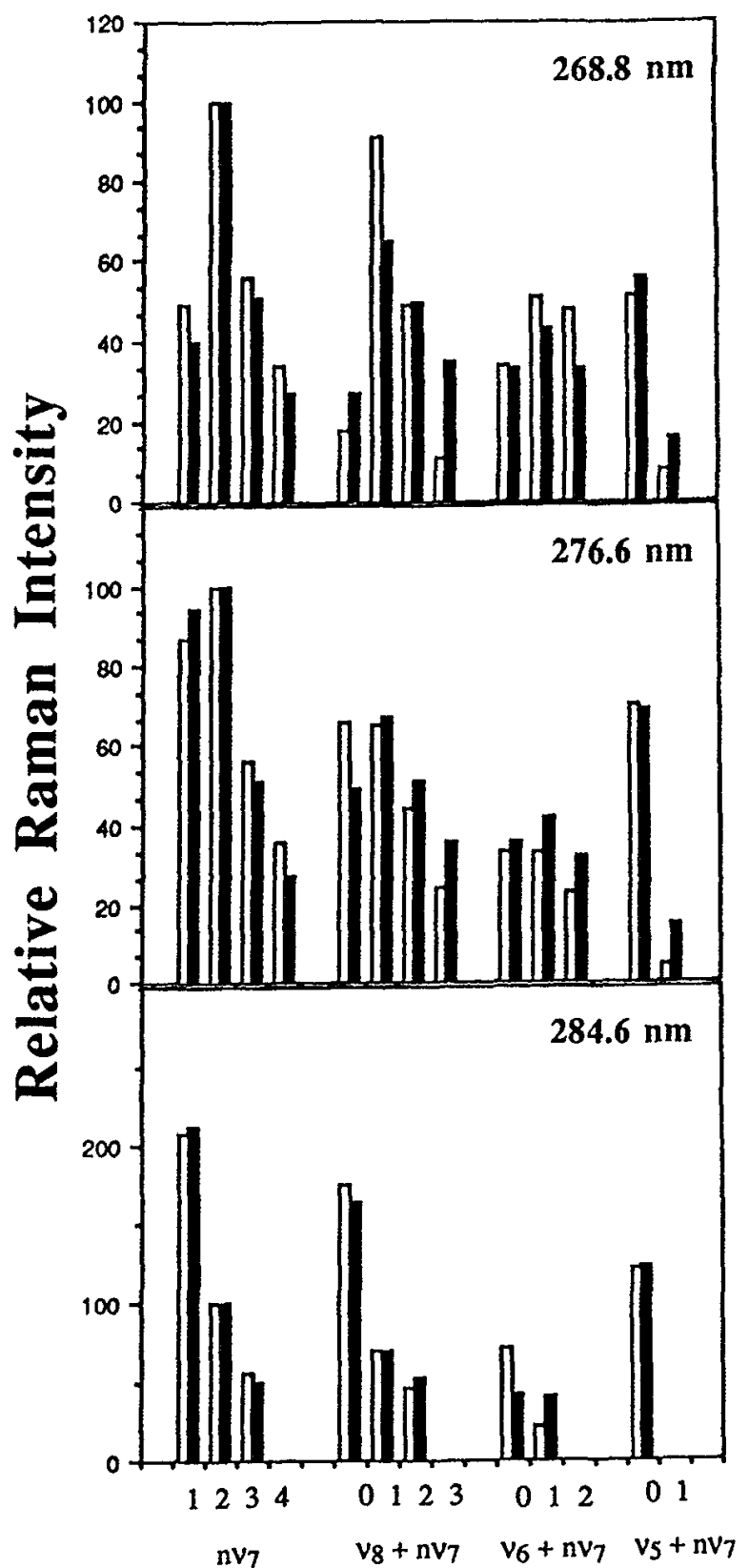

FIG. 11. Comparison of experimental (open bars) and calculated (solid bars) relative Raman intensities at three different excitation wavelengths in the $A$-band absorption of tert-butyl iodide in cyclohexane solution.

onstrates the importance of resonance-preresonance interferences in determining the observed fundamental intensities.

\section{E. Vibrational normal-mode calculations}

The ground-state normal modes of all three alkyl iodides were calculated by refining a valence force field starting from literature values to best-fit experimental vibrational frequencies of the parent molecules and the deuterated derivatives of ethyl iodide $\left(\mathrm{CD}_{3} \mathrm{CH}_{2} \mathrm{I}, \mathrm{CH}_{3} \mathrm{CD}_{2} \mathrm{I}\right.$, and $\left.\mathrm{CD}_{3} \mathrm{CD}_{2} \mathrm{I}\right)$ as described in Sec. III. The final force field gave $\mathrm{rms}$ frequency errors of $7.6 \mathrm{~cm}^{-1}$ for ethyl (all four isotopic derivatives), $2.9 \mathrm{~cm}^{-1}$ for isopropyl, and $7.6 \mathrm{~cm}^{-1}$ for tert- 


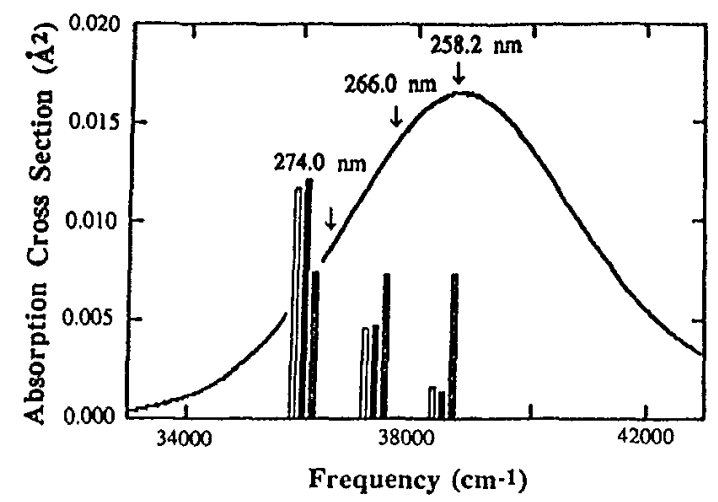

FIG. 12. Experimental ratio of the nominal C-I stretch fundamental to its first overtone $\left(v_{10} / 2 v_{11}\right)$ for ethyl iodide in cyclohexane solution (open bars), the ratio calculated including the nonresonant $A$-term contribution (solid bars), and the ratio calculated without the nonresonant contribution (striped bars). The absorption spectrum is shown for reference.

butyl iodide. Figure 13 shows the geometries assumed for the three alkyl iodides. The complete force fields, Cartesian coordinates, and computed vibrational frequencies and normal-mode coefficients are available as supplementary material. Table VIII summarizes the experimental and calculated vibrational frequencies and potential-energy distributions for the modes having significant intensity in the $A$-band resonant Raman spectra. The normal-mode descriptions show increased mixing of the $\mathrm{CI}$ stretching coordinate into the nominal CCI bending mode near $260 \mathrm{~cm}^{-1}$ as the alkyl group size is increased. However, most of the other modes that show resonance Raman intensity have little CI stretching component, indicating qualitatively that the initial photodissociation dynamics does involve significant motion along coordinates other than the CI stretch.

\section{F. Photodissociation dynamics}

With both the ground-state normal-mode descriptions and the excited-state dimensionless origin displacements in hand, we may now apply Eqs. (12) and (13) to calculate the change in the expectation value of each internal coordinate as a function of time following photoexcitation. Since we have used a harmonic potential to simulate a much more complex dissociative surface, we can justifiably discuss the dynamics only at short times, where the nuclei have not yet moved far from the Franck-Condon region. We choose $10 \mathrm{fs}$ as a reasonable time based on the observation that the overlaps $\langle f \mid 0(t)\rangle$ which determine the calculated resonance Raman intensities reach their maxima at times in the 5-10 fs range for most of the observed transitions. Also, $10 \mathrm{fs}$ is short compared with the recurrence times of any of the active vibrations.

At this point a complication arises. Since the fitting parameters of Table VII give only the absolute magnitude of each dimensionless displacement $\Delta$, we must, in general, consider all possible sign combinations, or $2^{n}$ different choices for $n$ Franck-Condon active normal modes. Imposing the physically reasonable requirement that the initial change in the $\mathrm{C}-\mathrm{I}$ bond length be in the positive direction
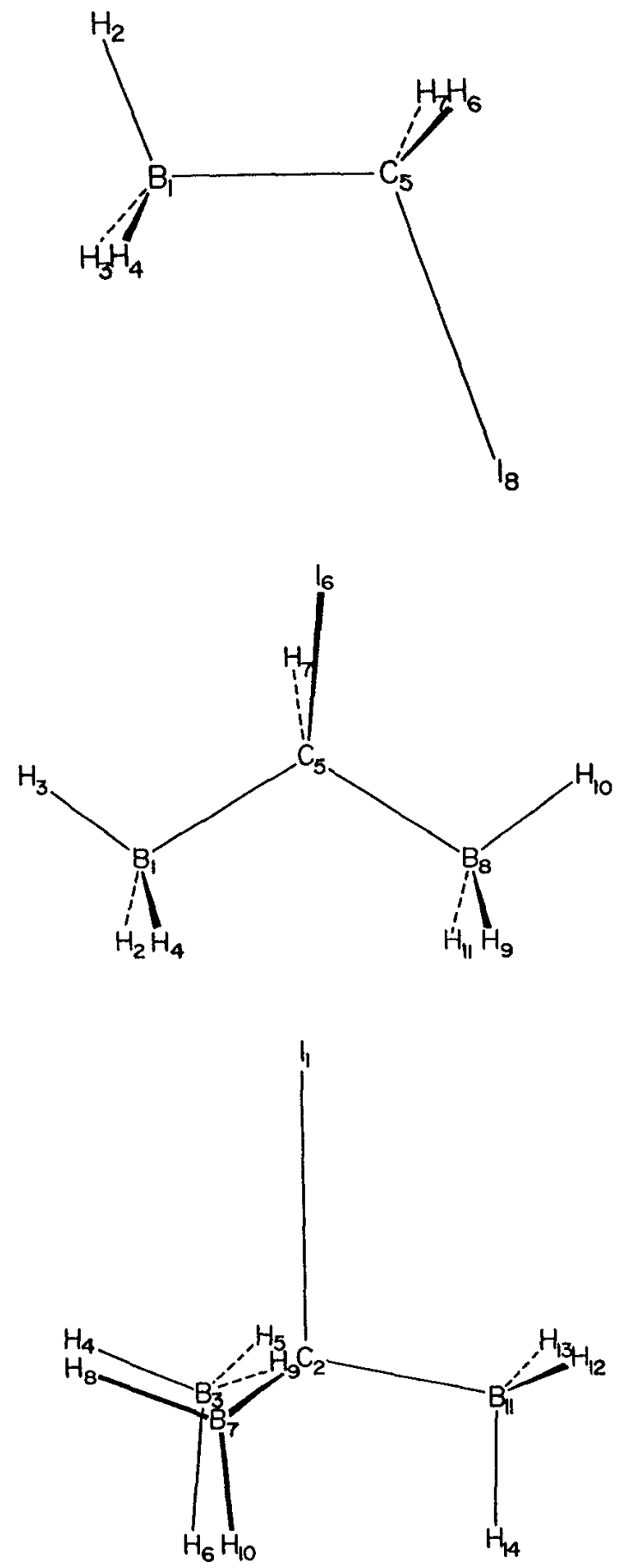

FIG. 13. Geometries of ethyl (top), isopropyl (middle), and tert-butyl (bottom) iodides used in the normal coordinate calculations. All $\mathrm{CH}$ bond lengths are $1.09 \AA, C C$ bond lengths are $1.54 \AA$, CI bond lengths are 2.135 $\AA$, and all bond angles are $109.5^{\circ}$. C represents the $\alpha$-carbon atom and $\mathrm{B}$ represents a methyl carbon atom.

eliminates only half these possibilities. Fortunately, for ethyl iodide we can further reduce the number of possibilities by making use of the redundancy contained in the Raman intensities of isotopic derivatives. ${ }^{23,51,52,61}$ Since the electronic structure problem is assumed to be isotope invariant, all isotopic derivatives should have the same geometry change upon electronic excitation (and, after accounting for mass differences, the same initial dynamics), but, in general, they 
TABLE VIII. Alkyl iodide ground-state normal modes.

\begin{tabular}{|c|c|c|c|}
\hline Mode & $\begin{array}{l}\text { Expt. freq. } \\
\left(\mathrm{cm}^{-1}\right)\end{array}$ & $\begin{array}{l}\text { Calc. freq. } \\
\left(\mathrm{cm}^{-1}\right)\end{array}$ & Calc. PEDa \\
\hline \multicolumn{4}{|c|}{ Ethyl } \\
\hline $\boldsymbol{v}_{11}$ & 262 & 260 & $63 \% \mathrm{BCI}, 19 \% \mathrm{CI}$ \\
\hline$v_{10}$ & 498 & 505 & $83 \% \mathrm{CI}, 20 \% \mathrm{BCI}$ \\
\hline$v_{8}$ & 1049 & 1053 & $57 \% \mathrm{CBH}, 19 \% \mathrm{BC}$ \\
\hline$v_{7}$ & 1203 & 1203 & $56 \% \mathrm{BCH}, 16 \% \mathrm{HCH}$ \\
\hline$v_{4}$ & 1450 & 1458 & $68 \% \mathrm{HBH}, 16 \% \mathrm{HCH}$ \\
\hline \multicolumn{4}{|c|}{ Perdeuterated ethyl } \\
\hline$v_{11}$ & 233 & 234 & $65 \% \mathrm{BCI}, 15 \% \mathrm{CI}$ \\
\hline$v_{10}$ & 458 & 451 & $81 \% \mathrm{CI}, 14 \% \mathrm{BCI}$ \\
\hline $\boldsymbol{v}_{7}$ & 972 & 973 & $\begin{array}{l}41 \% \text { BCH, } 24 \% \mathrm{HCI}, 22 \% \mathrm{CBH} \\
12 \% \mathrm{HBH}, 11 \% \mathrm{BC}\end{array}$ \\
\hline \multicolumn{4}{|c|}{ Isopropyl } \\
\hline $\boldsymbol{v}_{10}$ & 250 & 257 & $49 \% \mathrm{BCI}, 26 \% \mathrm{CI}, 17 \% \mathrm{CBH}, 13 \% \mathrm{BCB}$ \\
\hline$v_{8}$ & 499 & 496 & $71 \% \mathrm{CI}, 36 \% \mathrm{BCI}, 27 \% \mathrm{CBH}$ \\
\hline $\boldsymbol{v}_{7}$ & 879 & 877 & $48 \% \mathrm{BC}, 40 \% \mathrm{CBH}$ \\
\hline$v_{5}$ & 1153 & 1149 & $47 \% \mathrm{CBH}, 13 \% \mathrm{HBH}, 11 \% \mathrm{BC}$ \\
\hline$v_{4}$ & 1210 & 1211 & $35 \% \mathrm{CBH}, 27 \% \mathrm{HCI}, 17 \% \mathrm{BCH}$ \\
\hline \multicolumn{4}{|c|}{ Tert-butyl } \\
\hline$v_{8}$ & 259 & 246 & $52 \% \mathrm{CI}, 17 \% \mathrm{BCB}, 11 \% \mathrm{BCI}$ \\
\hline$v_{7}$ & 487 & 487 & $51 \%$ CI, $39 \%$ BCB, $24 \%$ BCI \\
\hline$v_{6}$ & 806 & 801 & $68 \% \mathrm{BC}, 17 \% \mathrm{CBH}$ \\
\hline$v_{3}$ & 1143 & 1157 & $63 \% \mathrm{CBH}, 11 \% \mathrm{HBH}$ \\
\hline
\end{tabular}

a Diagonal force constants contributing $10 \%$ or more to the total potential energy of the normal mode are given. $\mathrm{C}$ represents the $\alpha$-carbon atom and $\mathrm{B}$ represents a methyl carbon atom.

all have different normal-mode descriptions and therefore different resonance Raman intensities. (See Ref. 62 for a dramatic illustration of this.) Thus each possible choice of sign for each isotope generates a different excited-state geometry, and the one having the least variance across the set of derivatives is chosen as the most probable. In the present case we find that four of the $2^{n-1}$ possible excited-state geometries have rms differences between the $d_{5}$ and $h_{5}$ species that are much smaller (by factors of 2 or more) than any of the others, so we select these four as the only realistic possibilities. Table IX presents, for each significantly displaced coordinate, the range of internal coordinate displacements at 10 fs obtained from the dynamics calculated from these four (for ethyl) or $2^{n-1}$ (for isopropyl and tert-butyl iodides) different sign combinations. These results strongly suggest that significant changes in bond angles and $\mathrm{CC}$ bond lengths as well as elongation of the $\mathrm{CI}$ bond occur in the initial stages of alkyl iodide photodissociation. Unfortunately, the indeterminacy in the signs of the normal-mode displacements for isopropyl and tert-butyl iodides greatly limits the conclusions we can draw; not only can we not determine the magnitude of the initial geometry change with much precision, but we cannot even be definite about the sign of the displacements in most coordinates based on experimental data from a single isotopic derivative.

\section{DISCUSSION}

Determination of the signs of the normal-mode displacements obtained from vibronic analysis (absorption, fluorescence, or Raman) is a problem of long standing which has been addressed in a number of ways, none of them
TABLE IX. Internal coordinate displacements at $t=10 \mathrm{fs}$.

\begin{tabular}{|c|c|c|}
\hline & Coordinate $^{a}$ & Range of Displacements of $10 \mathrm{fs}^{\mathrm{b}}$ \\
\hline Ethyl & $\begin{array}{l}r_{\mathrm{Cl}} \\
r_{\mathrm{BC}} \\
\angle \mathrm{BCI} \\
\angle \mathrm{HCI} \\
\angle \mathrm{BCH}{ }_{9} \\
\angle \mathrm{H}_{6} \mathrm{CH}_{7} \\
\angle \mathrm{CBH}_{2} \\
\angle \mathrm{CBH}_{3} \\
\angle \mathrm{H}_{2} \mathrm{BH}_{3} \\
\angle \mathrm{H}_{2} \mathrm{BH}_{4}\end{array}$ & $\begin{array}{c}+0.13 /+0.15 \AA \\
-0.06 /-0.05 \AA \\
-6^{\circ} \%-3^{\circ} \\
-7^{\circ} /-3^{\circ} \\
+6^{\circ} /+9^{\circ} \\
-6^{\circ} \%+5^{\circ} \\
-1^{\circ} \%+5^{\circ} \\
-1^{\circ} \%-3^{\circ} \\
-4^{\circ} \%+5^{\circ} \\
-8^{\circ} \%+8^{\circ}\end{array}$ \\
\hline Isopropyl & $\begin{array}{l}r_{\mathrm{Cl}} \\
r_{\mathrm{BC}} \\
\angle \mathrm{BCI} \\
\angle \mathrm{BCB} \\
\angle \mathrm{H}_{7} \mathrm{CI} \\
\angle \mathrm{BCH}_{7} \\
\angle \mathrm{CBH}_{2} \\
\angle \mathrm{CBH}_{3} \\
\angle \mathrm{CBH}_{4}\end{array}$ & $\begin{array}{c}+0.06 /+0.15 \AA \\
-0.05 \%+0.05 \AA \\
-6^{\circ} \%-2^{\circ} \\
-2^{\circ} \%+5^{\circ} \\
-3^{\circ} \%+5^{\circ} \\
-1^{\circ} \%+6^{\circ} \\
-10^{\circ} \%+4^{\circ} \\
-10^{\circ} \%+5^{\circ} \\
-1^{\circ} \%+8^{\circ}\end{array}$ \\
\hline Tert-butyl & $\begin{array}{l}r_{\mathrm{CI}} \\
r_{\mathrm{BC}} \\
\angle \mathrm{BCI} \\
\angle \mathrm{BCB} \\
\angle \mathrm{CBH}{ }_{4}\end{array}$ & $\begin{array}{c}+0.02 /+0.17 \AA \\
-0.04 /+0.06 \AA \\
-6^{\circ} /-1^{\circ} \\
+1^{\circ} /+6^{\circ} \\
-4^{\circ} /+7^{\circ}\end{array}$ \\
\hline
\end{tabular}

${ }^{x}$ See Fig. 13 for atom numbering. $C$ represents the $\alpha$-carbon atom and $B$ represents a methyl carbon atom. Coordinates related by symmetry are omitted, as are coordinates having displacements of less than $0.01 \AA$ or $4^{\circ}$ in every set.

${ }^{b}$ Calculated from Eqs. (12) and (13) with best-fit normal-mode displacement parameters of Table VII; range encompasses best four sets of sign combinations for ethyl and all sets giving $r_{\mathrm{CI}}>0$ for isopropyl and tertbutyl (see text). 
fully satisfactory. In some cases the difficulty is skirted by simply assuming that the normal modes are pure internal coordinates (e.g., stretches), ${ }^{24,63}$ but this is not usually a valid assumption. Frequently, the geometry changes predicted by electronic structure calculations and/or other criteria based on chemical intuition can be used to eliminate many possible sign combinations. ${ }^{23,30,52,61,64}$ While useful, this approach does require prior knowledge about the excited state under study, and there can be considerable remaining ambiguity for large molecules. ${ }^{52}$ The sign problem may be solved, in principle, by incorporating intensity data for isotopic derivatives as we have done with ethyl iodide, but adequate definition of the problem for large molecules requires not only synthesis of many isotopic derivatives and extensive experimental work to obtain all the Raman spectra, but also highly accurate ground-state normal-mode descriptions. Since the number of independent force constants in the most general valence force field greatly exceeds the number of experimentally observed vibrational frequencies, the accuracy of the normal modes even within the harmonic approximation is always open to question. This is certainly a question in our ethyl iodide analysis in spite of the relatively small size of the molecule and the availability of frequencies for several isotopic derivatives. Finally, it should be noted that if one considers the complete harmonic problem including Duschinsky rotation, the relative signs of the displacements in the various normal modes do affect the computed intensities. It is sometimes possible to determine at least some of these relative signs from experimental data ${ }^{65}$ but rarely are the data adequate to unambiguously determine all the elements of the full Duschinsky matrix. Given these constraints, it would appear that the goal of fully determining excited-state geometries or dynamics from experimental data alone is not likely to be realized for any but a small class of simple molecules.

Yet in spite of these limitations, we can draw some definite conclusions about the initial dynamics of alkyl iodide photodissociation. Table IX shows that, for all three alkyl iodides, all sign combinations giving rise to the physically reasonable elongation of the $\mathrm{Cl}$ bond do have other points in common. The amount that the $\mathrm{CI}$ bond lengthens in the first $10 \mathrm{fs}$ is, not surprisingly, very similar in the three molecules studied $(0.13-0.15 \AA$ for ethyl iodide, with a somewhat greater variance across the set of possible sign combinations for the larger molecules). In all three molecules the CCI bond angle decreases by $1^{\circ}-8^{\circ}$ during the first $10 \mathrm{fs}$. This makes sense in terms of the evolution of the structure toward an alkyl radical that is more nearly planar about the carbon radical center, ${ }^{66,67}$ although it can also be viewed as simply a kinematic consequence of pushing the carbon atom into the alkyl fragment ("soft" radical limit) ${ }^{17}$ as the $\mathrm{CI}$ bond lengthens. Conclusions about the $\mathrm{CC}$ bond length changes are more difficult to reach from the data alone. Comparison of $a b$ initio structure calculations on the ethyl radical ${ }^{66,67}$ with the best available experimental geometry for ethyl iodide $^{57}$ suggests that the CC bond length is reduced in the radical relative to the parent molecule, and this is consistent with the results of our Raman intensity analysis which show a decrease of $0.05-0.06 \AA$ in the $\mathrm{C}-\mathrm{C}$ bond length during the first $10 \mathrm{fs}$. Our purely experimental results do not allow us to determine even the direction of the $\mathrm{CC}$ bond length changes in isopropyl and tert-butyl iodides.

Although the lack of isotopic data for isopropyl and tert-butyl iodides means that we are not able to reach firm conclusions about what most of the internal coordinates are doing in the photodissociation process, our results are inconsistent with any picture in which the initial motion involves $\mathrm{CI}$ bond breaking alone. Every individual set of sign combinations summarized in Table IX involves significant motion along several other coordinates, although these are different coordinates for different sign combinations. In particular, our fairly well-defined conclusions for ethyl iodide appear qualitatively inconsistent with studies of energy partitioning which suggest, based on the fraction of the available energy appearing as internal excitation of the separated photofragments, that the ethyl radical ends up with relatively little vibrational excitation ("rigid" radical limit) ${ }^{9,68,69}$ It should be noted that the internal energy of the ethyl radical obtained from translational photofragment spectroscopy is anomalous compared with all other alkyl iodides examined thus far. Ethyl iodide is the only one in which the energy partitioning approaches that predicted by the rigid radical model; in fact, the internal energy of the ethyl radical for the $I^{*}$ channel is even lower than that predicted in the rigid radical limit. Paterson, Godwin, and Gorry ${ }^{68}$ suggest that this could arise from changes in the geometry of the departing ethyl radical on the time scale of the bond breaking such that the torque imparted to the radical is reduced from the simple rigid radical value. Our results based on the resonance Raman intensities certainly support the idea that there is no clear separation of time scales between the C-I bond breaking and the vibrational motions of the remaining radical. These simple impulsive ("soft" and "rigid" radical) models for the photodissociation clearly involve significant approximations and are probably best considered as qualitative guides with which to compare experimental data. Finally, we note that the resonance Raman data indicate no significant coupling of $\mathrm{C}-\mathrm{H}$ stretching to the electronic excitation. This is consistent with the conclusion that the $\mathrm{H}$-atom formation observed upon alkyl iodide photolysis results from two-photon processes. ${ }^{70}$

It must be stressed that our results based on the resonance Raman intensities speak only to the very short-time dynamics, whereas the energy partitioning in the photofragments depends on the entire potential-energy surface out to the asymptotic limit. In principle, we can learn about progressively larger pieces of the potential surface by looking at Raman scattering to higher-lying ground-state vibrations, but this is simply not practical for these and most other medium-sized or larger molecules. The increased linewidths of the higher vibrational states, particularly in solution phase, coupled with the increasing density of Raman transitions having significant intensity, make the vibrational spectrum too congested to allow identification of individual vibrational lines at energies above a few thousand wave numbers. For this reason the resonance Raman technique must be considered complementary to other techniques, including translational photofragment spectroscopy, which probe the photo- 
dissociation products directly. Direct studies of the degree of vibrational excitation in the separated radical, such as antiStokes resonance Raman or infrared emission, will clearly be helpful in resolving these questions.

The analysis and discussion presented above has made little direct reference to the fact that these are solvated molecules. This reflects no lack of interest in or attention to possible effects of solvation, but rather that our data simply do not demonstrate much in the way of explicit solvent effects. The optical spectra of the alkyl iodides in cyclohexane are nearly identical to the vapor-phase spectra apart from a modest degree of solvent-induced broadening of the sharper bands. In particular, there is no evidence of optically allowed transitions to ionic or charge transfer to solvent states as are observed for $\mathrm{I}_{2}$ in certain solvents, ${ }^{71}$ although ionic dark states could perhaps play a role in the solution-phase photochemistry. The alkyl iodides, unlike molecules having bound or predissociative resonant electronic states, ${ }^{72}$ exhibit little or no broad fluorescence in their solution-phase emission spectra. This reflects the fact that photodissociation (motion of the wave packet out of the Franck-Condon region) on a steeply repulsive potential is faster than solvent-induced dephasing. There may be more subtle effects of solvation on the dissociation dynamics which will be revealed by a careful comparison of the solution- and vapor-phase resonance Raman spectra, although our preliminary results on methyl iodide $^{20}$ suggest that these are quite small, at least for the first tens of femtoseconds of dynamics to which the Raman intensities are principally sensitive. In contrast, in the linear polyenes we have resonance Raman evidence for large solvent effects on the short-time (tens of fs) dynamics of nuclear motion which may result from solvent-induced shifts in the relative positions of interacting potential-energy surfaces. ${ }^{73}$ The question of how both collisional and mean-field effects cause chemical reaction dynamics to differ between vapor and solution phases is certainly a very interesting and relevant one which further experimental and computational studies $^{74}$ will play important roles in addressing.

\section{ACKNOWLEDGMENTS}

This work was supported in part by a grant from the NSF (No. CHE-8709485). A.B.M. is the recipient of a Dreyfus Distinguished New Faculty Award, a Packard Fellowship in Science and Engineering, a Sloan Research Fellowship, and an NSF Presidential Young Investigator Award.

\footnotetext{
'M. Dzvonik, S. Yang, and R. Bersohn, J. Chem. Phys. 61, 4408 ( 1974). ${ }^{2}$ G. N. A. Van Veen, T. Baller, A. E. de Vries, and N. J. A. van Veen, Chem. Phys. 87, 405 (1984).

${ }^{3}$ F. G. Godwin, C. Paterson, and P. A. Gorry, Mol. Phys. 61, 827 (1987)

${ }^{4} \mathrm{~J}$. F. Black and I. Powis, Chem. Phys. 125, 375 (1988).

${ }^{5}$ P. Brewer, P. Das, G. Ondrey, and R. Bersohn, J. Chem. Phys. 79, 720 (1983).

'W. P. Hess, S. J. Kohler, H. K. Haugen, and S. R. Leone, J. Chem. Phys. 84, 2143 (1986).

${ }^{7} M$. Shapiro, J. Phys. Chem. 90, 3644 (1986).

${ }^{8}$ F. G. Godwin, P. A. Gorry, P. M. Hughes, D. Raybone, T. M. Watkinson, and J. C. Whitehead, Chem. Phys. Lett. 135, 163 (1987).
}

${ }^{9}$ Q. Zhu, J. R. Cao, Y. Wen, J. Zhang, X. Zhong, Y. Huang, W. Fang, and X. Wu, Chem. Phys. Lett. 144, 486 (1988).

${ }^{10}$ K. Q. Lao, M. D. Person, P. Xayariboun, and L. J. Butler, J. Chem. Phys. 92, 823 (1990).

"D. W. Chandler, M. H. M. Janssen, S. Stolte, R. N. Strickland, J. W. Thoman, Jr., and D. H. Parker, J. Phys. Chem. 94, 4839 (1990).

${ }^{12}$ H. Guo and G. C. Schatz, J. Chem. Phys. 93, 393 (1990).

${ }^{13} \mathrm{M}$. Shapiro and R. Bersohn, J. Chem. Phys. 73, 3810 (1980).

${ }^{14}$ L. R. Khundkar and A. H. Zewail, Chem. Phys. Lett. 142, 426 (1987).

${ }^{15}$ R. Ogorzalek Loo, H.-P. Haerri, G. E. Hall, and P. L. Houston, J. Chem. Phys. 90, 4222 (1989).

${ }^{16}$ R. L. Sundberg, D. Imre, M. O. Hale, J. L. Kinsey, and R. D. Coalson, J. Phys. Chem. 90, 5001 (1986).

${ }^{17}$ G. E. Busch and K. R. Wilson, J. Chem. Phys. 56, 3626 (1972).

${ }^{18}$ D. Imre, J. L. Kinsey, A. Sinha, and J. Krenos, J. Phys. Chem. 88, 3956 (1984).

${ }^{19}$ M. O. Hale, G. E. Galica, S. G. Glogover, and J. L. Kinsey, J. Phys. Chem. 90, 4997 (1986).

${ }^{20}$ F. Markel and A. B. Myers, Chem. Phys. Lett. 167, 175 (1990).

${ }^{21}$ A. Warshel, Annu. Rev. Biophys. Bioeng. 6, 273 (1977).

${ }^{22}$ D. C. Blazej and W. L. Peticolas, Proc. Natl. Acad. Sci. U.S.A. 74, 2639 (1977).

${ }^{23}$ P. G. Wright, P. Stein, J. M. Burke, and T. G. Spiro, J. Am. Chem. Soc. 101, 3531 (1979)

${ }^{24}$ R. J. H. Clark and B. Stewart, J. Am. Chem. Soc. 103, 6593 (1981).

${ }^{25}$ S.-Y. Lee and E. J. Heller, J. Chem. Phys. 71, 4777 (1979).

${ }^{26}$ W. Holzer, W. F. Murphy, and H. J. Bernstein, J. Chem. Phys. 52, 399 (1970).

${ }^{27}$ D. L. Rousseau and P. F. Williams, J. Chem. Phys. 64, 3519 (1976).

${ }^{28}$ G. Eyring, B. Curry, A. Broek, J. Lugtenburg, and R. Mathies, Biochem. 21, 384 (1982).

${ }^{29}$ A. B. Myers, R. A. Harris, and R. A. Mathies, J. Chem. Phys. 79, 603 (1983).

${ }^{30}$ A. B. Myers and R. A. Mathies, J. Chem. Phys. 81, 1552 (1984).

${ }^{31}$ (a) D. L. Phillips, B. A. Lawrence, and J. J. Valentini, J. Phys. Chem. (submitted); (b) See AIP document no. PAPS JCPSA-95-226-18 for 18 pages of tables: Order by PAPS number and journal reference from American Institute of Physics, Physics Auxiliary Publication Service, 335 East 45th Street, New York, NY 10017. The price is $\$ 1.50$ for each microfiche ( 98 pages) or $\$ 5.00$ for photocopies of up to 30 pages, and $\$ 0.15$ for each addiitonal page over 30 pages. Airmail additional. Make checks payable to the American Institute of Physics.

${ }^{32}$ P. Dawson, Spectrochim. Acta 28A, 715 (1972).

${ }^{33}$ A. B. Myers, B. Li, and X. Ci, J. Chem. Phys. 89, 1876 (1988).

${ }^{34}$ M. O. Trulson and R. A. Mathies, J. Chem. Phys. 84, 2068 (1986).

${ }^{35}$ B. Li and A. B. Myers, J. Phys. Chem. 94, 4051 (1990).

${ }^{30} \mathrm{~K}$. Kimura and S. Nagakura, Spectrochim. Acta 17, 166 (1961).

${ }^{37}$ D. J. Donaldson, V. Vaida, and R. Naaman, J. Chem. Phys. 87, 2522 (1987).

${ }^{38}$ P. Stein, V. Miskowski, W. H. Woodruff, J. P. Griffin, K. G. Werner, B. P. Gaber, and T. G. Spiro, J. Chem. Phys. 64, 2159 (1976).

${ }^{39}$ G. M. Korenowski, L. D. Ziegler, and A. C. Albrecht, J. Chem. Phys. 68, 1248 (1978).

${ }^{40}$ G. A. Schick and D. F. Bocian, J. Raman Spectrosc. 11, 27 (1981).

${ }^{41}$ S. A. Asher and C. R. Johnson, J. Phys. Chem. 89, 1375 (1985).

${ }^{42}$ S. P. A. Fodor, R. A. Copeland, C. A. Grygon, and T. G. Spiro, J. Am. Chem. Soc. 111, 5509 (1989).

${ }^{43}$ I. W. Sztainbuch and G. E. Leroi, J. Chem. Phys. 93, 4642 (1990).

${ }^{44}$ K.-S. K. Shin and J. I. Zink, J. Am. Chem. Soc. 112, 7148 (1990).

${ }^{45}$ A. C. Albrecht and M. C. Hutley, J. Chem. Phys. 55, 4438 (1971).

${ }^{46}$ A. B. Myers and R. A. Mathies, in Biological Applications of Raman Spectroscopy, edited by T. G. Spiro (Wiley, New York, 1987), Vol. 2, p. 1.

${ }^{47}$ A. Gedanken, Chem. Phys. Lett. 137, 462 (1987).

${ }^{48}$ R. J. Sension and H. L. Strauss, J. Chem. Phys. 85, 3791 (1986).

${ }^{49} \mathrm{O}$. Sonnich Mortensen and S. Hassing, Adv. Infrared Raman Spectrosc. 6, 1 (1980).

${ }^{50}$ E. J. Heller, R. L. Sundberg, and D. Tannor, J. Phys. Chem. 86, 1822 (1982).

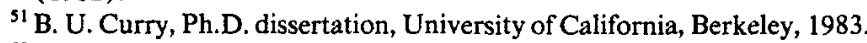

${ }^{52}$ A. B. Myers, Ph.D dissertation, University of California, Berkeley, 1984.

${ }^{53}$ E. B. Wilson, Jr., J. C. Decius, and P. C. Cross, Molecular Vibrations (Dover, New York, 1980).

${ }^{54}$ G. A. Crowder, J. Mol. Spectrosc. 48, 467 (1973).

${ }^{55}$ G. Crowder and Z. Najafi, Can. J. Chem. 55, 310 (1977). 
${ }^{50}$ W. Huttner and W. Zeil, Spectrochim. Acta 22, 1007 (1966).

${ }^{57}$ T. Inagusa, M. Fujitake, and M. Hayashi, J. Mol. Spectrosc. 128, 456 (1988).

${ }^{38}$ S. Felps, P. Hochmann, P. Brint, and S. P. McGlynn, J. Mol. Spectrosc. 59, 355 (1976).

${ }^{39} \mathrm{P}$. B. Kelly (personal communication).

${ }^{\infty 0}$ R. A. Boschi and D. R. Salahub, Mol. Phys. 24, 289 (1972); 24, 735 (1972).

${ }^{61}$ G. A. Schick and D. F. Bocian, J. Am. Chem. Soc. 106, 1682 (1984).

${ }^{62}$ L. C. Mayne and B. S. Hudson, J. Phys Chem. (submitted).

${ }^{63}$ K.-S. Shin, R. J. H. Clark, and J. I. Zink, J. Am. Chem. Soc. 111, 4244 (1989).

64 W. L. Peticolas and D. C. Blazej, Chem. Phys. Lett. 63, 604 (1979).

${ }^{69}$ A. B. Myers and K. S. Pranata, J. Phys. Chem. 93, 5079 (1989).
${ }^{66}$ B. H. Lengsfield III, P. E. M. Siegbahn, and B. Liu, J. Chem. Phys. 81, 710 (1984).

${ }^{67}$ J. Pacansky and B. Schrader, J. Chem. Phys. 78, 1033 (1983); J. Pacansky, W. Koch, and M. D. Miller, J. Am. Chem. Soc. 113, 317 (1991).

${ }^{68}$ C. Paterson, F. G. Godwin, and P. A. Gorry, Mol. Phys. 60, 729 (1987).

${ }^{69}$ S. J. Riley and K. R. Wilson, Faraday Discuss. Chem. Soc. 53, 132 (1972).

${ }^{70}$ J. L. Brum, S. Deshmukh, and B. Koplitz, J. Chem. Phys. 93, 7504 (1990); J. Am. Chem. Soc. 113, 1432 (1991).

${ }^{71}$ D. F. Evans, J. Chem. Phys. 23, 1424 (1955).

${ }^{72}$ A. B. Myers and B. Li, J. Chem. Phys. 92, 3310 (1990).

${ }^{73}$ X. Ci, M. A. Pereira, and A. B. Myers, J. Chem. Phys. 92, 4708 (1990).

${ }^{74}$ Y. S. Li and K. R. Wilson, J. Chem. Phys. 93, 8821 (1990). 